\title{
Photoionized Gas in Dark-Matter Minihalos in the Galactic Halo and Local Group
}

\author{
Orly Gnat and Amiel Sternberg \\ School of Physics and Astronomy and the Wise Observatory, The Beverly and Raymond \\ Sackler Faculty of Exact Sciences, Tel Aviv University, Tel Aviv 69978, Israel \\ orlyg@wise.tau.ac.il
}

\begin{abstract}
We present computations of the metals photoionization structures of pressure supported gas clouds in gravitationally dominant dark-matter mini-halos in the extended Galactic halo or Local Group environment. We consider low-metallicity (0.1 - 0.3 solar) clouds that are photoionized by the present-day metagalactic radiation field, and which are also pressure-confined by a hot external medium. We study the properties of $\Lambda$ CDM Burkert (and also NFW) mini-halos with characteristic circular velocities from 12 to $30 \mathrm{~km} \mathrm{~s}^{-1}\left(10^{8}-2 \times 10^{9} M_{\odot}\right)$. We present results for the volume densities and projected column densities of low-ionization metal atoms and ions, C II, N I, O I, Si II, and S II, produced in the neutral cloud cores, and high-ions, C IV, N V, O VI, Si III, Si IV, and S III produced in the ionized shielding envelopes. We examine the possible relationships between the compact H I high-velocity clouds (CHVCs), dwarf galaxies, and high-velocity ionized C IV absorbers, within the context of photoionized mini-halo models for such objects. In pressure-confined $\left(P / k \sim 50 \mathrm{~cm}^{-3} \mathrm{~K}\right)$ mini-halo models for the CHVCs the photoionization states are much lower than in the $\mathrm{C}$ IV absorbers. However, for lower bounding pressures $\lesssim 1 \mathrm{~cm}^{-3} \mathrm{~K}$, in more massive $\sim 2 \times 10^{9}$ $M_{\odot}$ dwarf galaxy scale halos, the photoionization states in the outer envelopes resemble those observed in the C IV HVCs. An important exception is O VI for which our photoionization models underpredict the relative abundances by about an order-of-magnitude, suggesting that additional processes are at play as concluded by Sembach et al. $(2000 ; 2003)$. For the cloud size-scales expected in median $\Lambda$ CDM Burkert halos, a gas metallicity of 0.3 solar is required to produce absorption-line strengths comparable to those in the C IV HVCs. We argue that fully ionized and starless "dark galaxies" could be detectable in the local universe as UV metal line absorbers with ionization states similar to the C IV absorbers.
\end{abstract}


Subject headings: dark matter - Galaxy:evolution - Galaxy:formation - Galaxy:general - intergalactic medium - Local Group - ISM:abundances - quasars:absorption lines

\section{Introduction}

Recent ultraviolet (UV) absorption-line observations have revealed the existence of highly ionized "high-velocity" gas clouds along lines of sight to extragalactic UV continuum sources (Sembach et al. 1999, 2000, 2002, 2003; Murphy et al. 2000; Wakker et al. 2003). These gas clouds may be the ionized components, or counterparts, of the well studied neutral hydrogen (H I) high-velocity clouds (HVCs) (Muller, Oort \& Raimond 1963; Wakker \& van Woerden 1997; Putman et al. 2002; de Heij, Braun \& Burton 2002), objects which are usually defined as $\mathrm{H}$ I systems with radial velocities inconsistent with differential rotation in the Galactic disk. The origin and nature of the HVCs remains controversial. One attractive possibility is that at least some of the high velocity gas, both neutral and ionized, represents remnant "infalling" material associated with galaxy formation processes (Oort 1970; Blitz et al. 1999; Braun \& Burton 1999; Sternberg, McKee \& Wolfire 2002).

In the UV studies, many high-ionization metal absorption lines have been detected, including Si III $\lambda$ 1206.5, Si IV $\lambda \lambda$ 1393.8, 1402.8, and C IV $\lambda \lambda$ 1548.2, 1550.8, observed with GHRS and STIS on board HST, and more recently O VI $\lambda 1031.9$ absorption observed with FUSE. Some of the ionized UV absorbers can, in fact, be identified with the large $\left(\sim 100 \mathrm{deg}^{2}\right)$ and nearby ( $\left.\lesssim 50 \mathrm{kpc}\right)$ H I HVC "complexes", such as the Magellanic Stream, or Complex C (Sembach et al. 2003). However, some absorbers, such as those observed towards Mrk 509 and PKS 2155-304 (Sembach et al. 1999; Collins, Shull \& Giroux 2004) do not appear directly linked to such structures, and could be clouds at much larger distances, perhaps distributed within the Local Group. The "high-ion absorbers" may be the local analogs of metal-line absorbers observed in damped Ly $\alpha$ systems at high redshifts (Lu et al. 1996; Prochaska \& Wolfe 1997; Boksenberg, Sargent \& Rauch 2003; Maller et al. 2003).

Sembach et al. (1999) suggested that photoionization by metagalactic radiation in low density clouds may be responsible for the C IV observed towards Mrk 509 and PKS 2155-304. However, the detection of highly ionized O VI along these sight-lines strongly suggests that additional processes, such as collisional ionization in conductive interfaces or in thermally unstable cooling gas, are important (Heckman et al. 2002; Fox et al. 2003).

In this paper we reexamine the properties of purely photoionized clouds, within the 
context of dark-matter "mini-halo" models for the compact H I high-velocity clouds (CHVCs) and dwarf galaxies. The CHVCs are of particular interest. They are kinematically distinct objects, with typical angular diameters $\lesssim 1^{\circ}$ as determined by the recent HIPASS and Leiden-Dwingeloo $21 \mathrm{~cm}$ surveys (Putman et al. 2002; de Heij et al. 2002), and they may be at larger characteristic distances from the Galaxy disc than are the large HVC complexes. In Sternberg, McKee \& Wolfire (2002; hereafter SMW) we explored the hypothesis (Blitz et al. 1999; Braun \& Burton 1999) that the population of CHVCs traces dark-matter (DM) substructure (Moore et al. 1999; Klypin et al. 1999) in the Galactic halo or in the more extended Local Group environment. In this picture the CHVCs are essentially the lower-mass "cousins" of the Local Group dwarf galaxies, and represent objects in which gas is confined to low-mass dark-matter "mini-halos". In SMW we focused on the phase properties of hydrogen gas, ionized/neutral/warm/cold, of hydrostatic gas clouds in gravitationally dominant DM halos, including the effects of photoionization and heating by the metagalactic radiation field, and pressure confinement by an external hot medium. Explicit models were constructed for the observed distributions of warm H I in the Local Group galaxies Leo A and Sag DIG. The H I boundaries in these objects are consistent with a cutoff due to photoionization by the metagalactic field.

The aim of this paper is to study the associated metals photoionization structures and resulting absorption-line properties of such $\left(10^{8}-2 \times 10^{9} M_{\odot}\right)$ mini-halo clouds. We consider photoionization by the present-day metagalactic field, which is the dominant source of radiation at distances $\gtrsim 150 \mathrm{kpc}$ from the Galactic disk (SMW). The calculations and models we construct are similar to those presented by Kepner et al. (1999) in their mini-halo models for the QSO absorption-line systems. Our models are more directly applicable to CHVCs in the outer Galactic halo and dwarf galaxies in the Local Group. In particular, we determine the conditions required for the formation of highly ionized absorbers similar to the C IV HVCs.

In $\S 2$ we discuss the basic parameters of our mini-halo models, and describe the methods we used to compute the metals photoionization structure. In $\S 3$ we present auxiliary computations of the abundances of highly ionized metal species in uniform density and optically thin clouds that are photoionized by the present-day metagalactic radiation field. In $\S 4$ we present photoionization calculations for mini-halo clouds for a range of dark-matter and gas masses, metallicities, radiation fields and bounding gas pressures. We compare our results to the C IV absorbers towards Mrk 509 and PKS 2155-304. We summarize in $§ 5$. 


\section{Model Ingredients}

We are interested in studying the metals photoionization structures of mini-halo clouds similar to those considered by SMW in their analysis of the H I CHVCs and Local Group dwarf galaxies. Following SMW, we construct models for warm, $T=10^{4} \mathrm{~K}$, pressure supported, spherically symmetric clouds that are; (a) in hydrostatic equilibrium in gravitationally dominant dark-matter halos, (b) photoionized by the present-day metagalactic radiation field, and (c) subjected to an external bounding pressure provided by an external hot ionized medium (HIM). The clouds typically consist of "warm neutral medium" (WNM) cores, surrounded by "warm ionized medium" (WIM) shielding envelopes that absorb the incident photoionizing radiation. The WNM/WIM mass ratios depend on several quantities, including the total (ionized plus neutral) warm cloud masses $M_{\mathrm{WM}}$, the depths of the dark-matter gravitational potential wells, the bounding HIM pressures, and the intensity of the ionizing radiation. In SMW we determined the conditions required for the formation of multi-phased mixtures of "cold neutral medium" (CNM) condensations together with the WNM in the cloud cores. In this paper we present calculations of the metals photoionization structures. We use our models to compute the distributions and column densities of metal ions in minihalo clouds for a range of conditions.

For the dark-matter densities, we focus mainly on halos with the empirically based Burkert (1995) profiles

$$
\rho_{\mathrm{DM}}=\frac{\rho_{d s}}{(1+x)\left(1+x^{2}\right)}
$$

where $x \equiv r / r_{s}$ is the radial distance from the halo center measured relative to the scale radius $r_{s}$, and $\rho_{d s}$ is the characteristic dark-matter scale density. We also consider one NFW model (Navarro, Frenk, \& White 1995), for which

$$
\rho_{\mathrm{DM}}=\frac{\rho_{d s}}{x(1+x)^{2}}
$$

Cosmological simulations and observations suggest that in realistic halos the scale parameters

$r_{s}$ and $\rho_{d s}{ }^{1}$ are correlated. We adopt a $\Lambda \operatorname{CDM}\left(H_{0}=0.70 \mathrm{~km} \mathrm{~s}^{-1} \mathrm{Mpc}^{-1}, \Omega_{m}=0.3, \Omega_{\Lambda}=0.7\right.$ and $\left.\sigma_{8}=0.9\right)$ correlation as specified by the formula

$$
x_{\mathrm{vir}}=27 \times 10^{0.14 \sigma}\left(\frac{M_{\mathrm{vir}}}{10^{9} M_{\odot}}\right)^{-0.08}
$$

that relates the concentration parameter $x_{\mathrm{vir}} \equiv r_{\mathrm{vir}} / r_{s}$ (where $r_{\mathrm{vir}}$ is the virial radius) and virial mass $M_{\text {vir }}$, for low-mass halos (Bullock et al. 2001; SMW; Wechsler et al. 2002). In

\footnotetext{
${ }^{1}$ In Burkert halos, $\rho_{d s}$ is the dark-matter density at $x=0$. For NFW halos $\rho_{d s}$ is the density at $x=0.46$.
} 
this expression $\sigma$ is the standard deviation for underconcentrated or overconcentrated halos. Given $M_{\text {vir }}$ and $x_{\text {vir }}$, the scale density and radius are given by $\rho_{d s}=\Delta \rho_{u} x_{\text {vir }}^{3} / f_{M}\left(x_{\text {vir }}\right)$ and $r_{s}=\left(3 / 4 \pi \Delta \rho_{u}\right)^{1 / 3} M_{\text {vir }}^{1 / 3} / x_{\text {vir }}$, where $\rho_{u}$ is the mean cosmic matter density, $\Delta \sim 340$ is the mean overdensity in virialized halos, and $f_{M}\left(x_{\mathrm{vir}}\right)$ is the dimensionless DM mass enclosed within the virial radius (SMW). The characteristic halo scale velocity is defined by $v_{s}^{2} \equiv(4 \pi G / 3) \rho_{d s} r_{s}^{2}$.

For the (isotropic) metagalactic radiation field we consider two options. First, we assume a "standard field" as given by the SMW fit

$$
J_{\nu}= \begin{cases}1.051 \times 10^{2} J_{\nu 0}\left(\frac{\nu}{\nu_{0}}\right)^{-1.5} & , \frac{\nu}{\nu_{0}}<0.3 \\ J_{\nu 0}\left(\frac{\nu}{\nu_{0}}\right)^{-5.41} & , \quad 0.3<\frac{\nu}{\nu_{0}}<1 \\ J_{\nu 0}\left(\frac{\nu}{\nu_{0}}\right)^{-3.13} & , 1<\frac{\nu}{\nu_{0}}<4 \\ 2.512 \times 10^{-2} J_{\nu 0}\left(\frac{\nu}{\nu_{0}}\right)^{-0.46} & , 4<\frac{\nu}{\nu_{0}}\end{cases}
$$

for the $1.6 \mu \mathrm{m}$ to $3 \mathrm{keV}$ range. In this expression $J_{\nu 0}=2 \times 10^{-23} \mathrm{erg} \mathrm{s}^{-1} \mathrm{~cm}^{-2} \mathrm{~Hz}^{-1} \mathrm{sr}^{-1}$, and $\nu_{0}$ is the Lyman limit frequency. This fit, which we plot in Figure 1, is based on observational constraints for the optical/UV and X-rays (Bernstein, Freedman \& Madore 2002; Martin, Hurwitz \& Bowyer 1991; Chen, Fabian, \& Gendreau 1997). For the unobservable radiation from the Lyman limit to $\sim 0.25 \mathrm{keV}$ it is based on theoretical models (Haardt \& Madau 1996; see also Shull et al. 1999). Second, we consider a "maximal field" that is enhanced between the Lyman limit and $0.25 \mathrm{keV}$ as illustrated by the dashed line in Figure 1 . For photon energies above the Lyman limit our maximal field is represented by

$$
J_{\nu}= \begin{cases}J_{\nu 0}\left(\frac{\nu}{\nu_{0}}\right)^{-1.725} & , 1<\frac{\nu}{\nu_{0}}<18.4 \\ 2.512 \times 10^{-2} J_{\nu 0}\left(\frac{\nu}{\nu_{0}}\right)^{-0.46} & , 18.4<\frac{\nu}{\nu_{0}}\end{cases}
$$

It is identical to the standard field in the optical/UV and X-rays, except for the enhancement from the Lyman limit to $\sim 0.25 \mathrm{keV}$. The Lyman continuum photon intensities implied by expressions (4) and (5) are both consistent with measured upper-limits on the $\mathrm{H} \alpha$ surface brightnesses of intergalactic clouds (Vogel et al. 1995; Weiner et al. 2001).

In our numerical computations, we begin by calculating the coupled hydrostatic and ionization structures of pure hydrogen-helium mini-halo clouds, assuming a primordial helium abundance $n_{\mathrm{He}} / n_{\mathrm{H}}=1 / 12$, and following the iterative radiative transfer procedures outlined by SMW. For the range of metallicities ( 0.1 to 0.3 times solar) and total hydrogen column densities ( up to $\sim 10^{21} \mathrm{~cm}^{-2}$ ) that we consider, the opacity is dominated by the hydrogen and helium absorptions, and metals are negligible. Dust opacity may also be ignored, assuming Galactic dust properties, and a dust to gas ratio proportional to the metallicity ${ }^{2}$.

\footnotetext{
${ }^{2}$ At $1000 \AA$ the dust absorption cross section per hydrogen nucleus $\sigma=1.9 \times 10^{-21} \mathrm{~cm}^{-2}$ for $\mathrm{Galactic}^{-}$
} 
The result of these calculations is the density and ionization profiles of the hydrogen-helium gas, extending from the halo center to an outer cloud radius, $r=r_{\mathrm{W} / \mathrm{H}}$, where the WIM gas pressure equals the bounding HIM pressure.

Given the H I density profile we then calculate the local absorbed radiation field

$$
J_{\nu}(r)=\frac{1}{2} \int_{1}^{-1} J_{\nu}^{0} e^{-\sigma(\nu) N_{H I}(r, \mu)} d \mu
$$

where in this expression $J_{\nu}^{0}$ is the unattenuated field, $\sigma(\nu)=\sigma_{H}(\nu)+\sigma_{H e}(\nu) / 12$ is the hydrogen-helium photo-absorption cross-section, $N_{\mathrm{HI}}(r, \mu)$ is the $\mathrm{H}$ I column density from $r$ to the cloud surface along a ray inclined at an angle $\theta$ relative to the radial direction, and where $\mu=\cos \theta$.

Finally, given the local hydrostatic hydrogen gas density, $n_{\mathrm{H}}(r)$, and the previously computed local absorbed radiation field, $J_{\nu}(r)$, we use the photoionization code CLOUDY (version 94.00, Ferland et al. $1998^{3}$ ) to calculate the local equilibrium ionization states for the metals. In this procedure we typically divide the clouds into up to 800 optically thin shells. For each thin shell we call CLOUDY using the local absorbed field computed in our prior radiative transfer computations. We assume isothermal $10^{4} \mathrm{~K}$ gas ${ }^{4}$, and fixed metallicity independent of radius. Given the run of ion volume densities, we then compute the line-ofsight ion column densities, $N_{\text {ion }}(b)$, where $b$ is the projected distance of the line-of-sight from the cloud center.

In $\S 4$ we present models for a range of conditions. We consider mini-halos with virial masses from $10^{8}$ to $2 \times 10^{9} M_{\odot}$, as appropriate for the CHVC to dwarf galaxy mass range. We consider total warm cloud gas masses (ionized plus neutral) from $10^{6}$ to $10^{9} M_{\odot}$, with HIM bounding pressures ranging from 0.01 to $50 \mathrm{~cm}^{-3} \mathrm{~K}$. Our models indicate how the metal absorption-line properties depend on these parameters, as well as the ionizing field, and metallicity.

dust (Draine 2003). Assuming that the absorption is proportional to the metallicity, a dust optical depth of $\tau=0.86$ is expected for $N_{\mathrm{H}}=1.5 \times 10^{21} \mathrm{~cm}^{-2}$ and a metallicity of 0.3 .

${ }^{3}$ See http://www.nublado.org

${ }^{4}$ Our assumed gas temperature is consistent with explicit CLOUDY computations of the thermal balance, showing that $T$ lies in the range of $8 \times 10^{3}$ to $3 \times 10^{4}$ for the WIM densities $\left(\lesssim 10^{-2} \mathrm{~cm}^{-3}\right)$ and range of metallicities we consider. 


\section{Photoionization by the Metagalactic Field}

Before we discuss our mini-halo cloud models, we present auxiliary computations for the ionization states of uniform density and optically thin gas that is photoionized by the metagalactic field. These auxiliary computations will be useful in understanding the results of our mini-halo cloud models in which the gas density varies, and in which optical depth effects are important. Here we consider individual parcels of $10^{4} \mathrm{~K}$ gas with hydrogen densities $n_{\mathrm{H}}$ ranging from $10^{-7}$ to $1 \mathrm{~cm}^{-3}$. This range corresponds to cosmological overdensities $\delta$ ranging from $\sim 1$ to $4 \times 10^{6}$, relative to the present-day mean baryon density $n_{b}=2.5 \times 10^{-7} \mathrm{~cm}^{-3}$ (for $\Omega_{b}=0.04$, Spergel et al. 2003). We assume a metallicity of 0.1 times the solar abundances as given by Holweger (2001). We list our 0.1 solar metal abundances in Table 1.

In Figure 2 we plot ${ }^{5}$, as functions of $n_{\mathrm{H}}$ and for our standard field, the relative and absolute densities of the various "high-ions", C IV, N V, O VI, Si III, Si IV, and S III, that we study in $\S 4$. We define high-ions as those with ionization thresholds greater than the hydrogen photoionization threshold of $13.6 \mathrm{eV}$. These will generally be present mainly in the WIM envelopes of our mini-halo clouds. The upper panel shows the fractional ion abundances, $n_{\text {ion }} / n_{\mathrm{H}}$, versus $n_{\mathrm{H}}$. The lower panel shows the absolute ion densities $n_{\text {ion }}$ versus $n_{\mathrm{H}}$. Because we have assumed optically thin conditions, and fixed temperature, both the relative and absolute ion densities scale linearly with the metallicity.

The "optimal" hydrogen density required to produce a particular ion depends on the ionization parameter

$$
U \equiv \frac{4 \pi J^{*}}{n_{\mathrm{H}} c}
$$

where the Lyc integral

$$
4 \pi J^{*}=\int d \Omega \int_{\nu_{0}}^{\infty} d \nu \frac{J_{\nu}}{h \nu},
$$

and $c$ is the speed of light. For our standard and maximal fields, $4 \pi J^{*}$ equals $1.3 \times 10^{4}$ and $2.2 \times 10^{4}$ photons $\mathrm{s}^{-1} \mathrm{~cm}^{-2}$ respectively. The gas becomes more highly ionized when $U$ is large. Thus, in Figure 2 where the radiation field is held constant, the ionization state increases with decreasing gas density, as $U$ varies from $4.3 \times 10^{-7}$ to 13.6 . The order of the various peaks in Figure 2a, is consistent with the photon energies required to produce the ions, O VI (113.90eV), N V (77.47eV), C IV (47.89eV), Si IV (33.49eV), S III (23.33eV), and Si III $(16.35 \mathrm{eV})$. Figure 2a shows that for our standard field the fractional abundances of O VI, C IV, and Si III are maximized at hydrogen gas densities of $3 \times 10^{-6}, 3 \times 10^{-5}$, and

\footnotetext{
${ }^{5}$ Color versions of this and other figures appearing in this paper are available in the electronic on-line edition.
} 
$6 \times 10^{-4} \mathrm{~cm}^{-3}$ respectively. For the maximal field the optimal densities are a factor $\sim 2$ larger.

The absolute ion density is also an important quantity. For a given length scale the ion column densities are largest when the ion densities approach the maximal values indicated in Fig. 2b. In our mini-halo models, for example, the path-lengths are set by the cloud sizes and the ion columns are largest along lines-of-sight that intersect the locations where the ion densities are largest (see equation[10] in $\S 4$ ). It is evident from Fig. 2 that the locations of the density maxima do not necessarily coincide with the fractional abundances maxima. The behavior is governed by how rapidly the ionization efficiency varies with density. For example, the O VI and C IV densities peak close to the locations of the fractional abundances maxima. In contrast, the Si III density continues to grow as $n_{\mathrm{H}}$ is increased above the density of $6 \times 10^{-4} \mathrm{~cm}^{-3}$ at which the Si III fractional abundance is maximized. The maximum C IV density occurs at $n_{\mathrm{H}}=1 \times 10^{-4} \mathrm{~cm}^{-3}$. For $T=10^{4} \mathrm{~K}$ this corresponds to a gas pressure $P / k \sim 2 \mathrm{~cm}^{-3} \mathrm{~K}^{6}$. At this pressure and density the expected $\mathrm{C}$ IV density in the metagalactic field is $\sim 7 \times 10^{-10} \mathrm{~cm}^{-3}$, for a metallicity of 0.1 .

Figure 2 shows the dependence of the ionization state versus hydrogen density for an unattenuated field. In the mini-halo models we discuss next, the results displayed in Figure 2 are directly applicable to the outer WIM layers where the gas is close to the optically thin limit. They become less accurate with increasing cloud depth, as the radiation field is absorbed and the various ionization rates are selectively diminished.

\section{Mini-halo models}

Our goal is to determine the metal column density distributions and UV absorption line properties of gas clouds in low-mass $\left(10^{8}-2 \times 10^{9} M_{\odot}\right)$ mini-halos that are photoionized by the present-day metagalactic field. In particular, we wish to find conditions that yield metal ion column densities comparable to those observed in the high-velocity C IV absorbers. As a guide to these observations, we focus on the results presented by Sembach et al. $(1999 ; 2000)$ and Collins et al. (2004) for the C IV HVCs towards the extragalactic sources Mrk 509 and PKS 2155-304. In Table 2 we list the low-ionization C II, N I, Si II, S II, and high-ionization C IV, N V, Si III, Si IV, and S III column densities inferred by Sembach et al. (1999) for each of two high-velocity components observed towards each line of sight. Sembach et al. (2000; 2003) detected O VI absorption along these lines-of-sight, and we include the inferred O VI columns in Table 2. More recently, Collins et al. (2004) have presented new data on

\footnotetext{
${ }^{6}$ For our assumed helium abundance $P=2.17 n_{\mathrm{H}} k T$ for fully ionized gas.
} 
the Mrk 509 and PKS 2155-304 absorbers, including observations obtained with HST STIS. We summarize the Collins et al. data in Table 3.

We present two classes of illustrative models. First, we analyze the photoionization structures of the low-mass $\left(\sim 10^{8} M_{\odot}\right)$, pressure-confined, mini-halo clouds that were presented by SMW as representative models for the H I CHVCs. We recall that these two models were constructed by SMW to match the observed CHVC H I thermal phase properties, assuming "circumgalactic" or "extragalactic" distances of 150 or $750 \mathrm{kpc}$ for these objects. The characteristic mass-scale for the CHVC mini-halos is set by the number of such objects $(\gtrsim 200)$ in comparison with theoretical simulations that predict a steeply rising mass-spectrum towards low mass objects in the dark-matter substructure (Moore et al. 1999; Klypin et al. 1999; SMW). As we will show, the assumed external pressures $\left(P_{\mathrm{HIM}} / k \sim 50\right.$ $\mathrm{cm}^{-3} \mathrm{~K}$ ) in these models severely limit the abundances of the high ions produced by photoionization in the WIM envelopes. Second, we consider gravitationally-confined clouds in more massive $\left(2 \times 10^{9} M_{\odot}\right)$ "dwarf-galaxy" scale halos, in which enhanced gravitational binding of the gas allows for lower external confining pressures and hence increased ionization in the WIM.

In Table 4 we list the basic input parameters for the various models. These include, the halo virial mass $M_{\text {vir }}$, concentration parameter $x_{\text {vir }}$, scale radius and velocity, $r_{s}$ and $v_{s}$, radiation field (standard or maximal), total gas mass $M_{\mathrm{WM}}$ (ionized plus neutral), metallicity, and external HIM pressure $P_{\text {HIM }}$.

The parameters we have chosen for the two pressure-confined systems are identical to those set by SMW for the CHVCs as circumgalactic or extragalactic objects. We refer to these as models A and B. The circumgalactic CHVC (model A) is a median $(\sigma=0)$ Burkert halo with $M_{\text {vir }}=1.0 \times 10^{8} M_{\odot}$ and $x_{\text {vir }}=32.5$, containing a total gas mass $M_{\mathrm{WM}}=1.1 \times 10^{6} M_{\odot}$ with 0.1 solar metallicity, exposed to the standard metagalactic field, and subjected to an external pressure $P_{\mathrm{HIM}} / k=50 \mathrm{~cm}^{-3} \mathrm{~K}$. SMW invoked a hot $2 \times 10^{6} \mathrm{~K}$ Galactic corona as the source of the required HIM pressure at $150 \mathrm{kpc}$. The extragalactic CHVC (model B) is a $-4 \sigma$ underconcentrated $3.2 \times 10^{8} M_{\odot}$ Burkert halo with $x_{\text {vir }}=8.2, M_{\mathrm{WM}}=7.1 \times 10^{7} M_{\odot}$, again with 0.1 solar metallicity and exposed to the standard field, with an assumed IGM bounding pressure $P_{\mathrm{HIM}}=20 \mathrm{~cm}^{-3} \mathrm{~K}$. Both models were constructed such that the resulting peak H I column densities are within the observed range of $2 \times 10^{19}$ to $2 \times 10^{20} \mathrm{~cm}^{-2}$ for the CHVCs. The relatively high external pressures in these models ensures that, (a) the gas remains bound ${ }^{7}$ to the halos at all cloud radii, and that (b) the gas is compressed to

\footnotetext{
${ }^{7}$ We define particles as "bound" if their gravitational potential energies exceed their mean thermal energies.
} 
densities that allow the formation of neutral hydrogen cores (which are then detectable as 21 cm sources). For such low-mass halos, the gas becomes ionized and then unbound as $P_{\text {HIM }}$ is reduced.

Figures 3 and 4 show the hydrogen and metal ion density distributions and projected column densities for our pressure-confined models. In model A, the neutral WNM gas core extends to a radius $r_{\mathrm{WNM}}=0.67 \mathrm{kpc}$, and the WIM shielding envelope extends to an outer cloud radius $r_{\mathrm{W} / \mathrm{H}}=1.3 \mathrm{kpc}$. In model $\mathrm{B}, r_{\mathrm{WNM}}=3.4 \mathrm{kpc}$, and $r_{\mathrm{W} / \mathrm{H}}=7.2 \mathrm{kpc}$. The low-ions, C II, N I, O I, Si II, and S II, are the dominant ionization stages in the WNM. The high-ions, C IV, N V, O VI, Si III, Si IV, and S III, are produced primarily in the WIM envelopes. These are general properties of all of the models in our parameter space. In the WNM, carbon, silicon, and sulfur are maintained in singly ionized form, while nitrogen and oxygen, with ionization potentials only slightly greater than $13.6 \mathrm{eV}$, remain neutral. In the WNM, the densities of the low-ions are simply proportional to the total hydrogen gas density, and are equal to the total metal abundances. The column densities of the low-ions are proportional to the line-of-sight H I columns through the WNM. Observations of low-ions along lines-of-sight through such H I WNM cores may therefore provide direct measures of the metallicity when photoionization is the dominant ionization mechanism in the cores.

For the Burkert halos we are considering, the low-ionization metal columns at $r=0$ may be approximated by

$$
N_{\text {low }}=x_{\text {metal }} N_{\mathrm{HI}, 0} \simeq \sqrt{\pi} x_{\text {metal }} n_{\mathrm{H}, 0} r_{\text {gas }}
$$

where $N_{\mathrm{HI}, 0}$ is the central (peak) H I column, $x_{\text {metal }}$ is the metal abundance, $n_{\mathrm{H}, 0}$ is the hydrogen gas density at the cloud center, and $r_{\text {gas }}$ is the $1 / e$ gas scale height for the neutral gas $^{8}$. For example, in Figure $3, N_{\mathrm{HI}, 0}=5 \times 10^{19} \mathrm{~cm}^{-2}$ and $N_{\mathrm{OI}, 0}=3 \times 10^{15} \mathrm{~cm}^{-2}$, consistent with our assumed oxygen abundance of $5.4 \times 10^{-5}$. The column densities of the low-ions in models $\mathrm{A}$ and $\mathrm{B}$ are comparable because the $\mathrm{H}$ I columns in these models are approximately the same by construction.

In both models A and B, C II, Si II, and S II remain the dominant stages in the WIM. However, N I and O I decrease sharply beyond the ionization fronts and into the WIM where these neutral stages are efficiently removed by photoionization. The densities of the high-ions produced in the WIM are controlled by several competing effects. First, the ionization state

\footnotetext{
${ }^{8}$ The relation $N_{\mathrm{HI}, 0}=\sqrt{\pi} n_{\mathrm{H}, 0} r_{\text {gas }}$ for Burkert halos is exact in the gravitationally-confined limit which obtains when $v_{s} / c_{g} \gtrsim 2$, where $c_{g}$ is the sound speed. In this limit $r_{\text {gas }}=0.44 \times 10^{-0.175 \sigma} c_{g 6} M_{\mathrm{vir}, 8}^{0.100} \mathrm{kpc}$, where $c_{g 6}$ is the sound speed in units of $10 \mathrm{~km} \mathrm{~s}^{-1}$, and $M_{\mathrm{vir}, 8}$ is the virial mass in units of $10^{8} \mathrm{M}_{\odot}$. These expressions are accurate to within a factor of $\sim 2$ for $v_{s} / c_{g}=1.5$ as is the case for our CHVC models (see SMW for a discussion of these analytic expressions).
} 
of the gas is largest near the cloud edges, where the ionization parameter is largest, i.e., where the gas density is lowest and where the radiation field is least attenuated. But second, the absolute ion densities may be maximized in inner regions where the overall gas densities are larger, or where the ionization parameter is optimized for the production of the particular ion. Third, the absorption of the ionizing radiation, especially near the ionization front, modifies the ionization structure. Figure $2 \mathrm{~b}$ may be used as a guide to the gas densities and cloud radii at which the density of a particular high-ion may be expected to be most efficiently produced, neglecting optical depth effects. For example, in models A and B the C IV, Si III, and Si IV densities are largest at the cloud edge. In model A, where $n_{\mathrm{H}}=2.3 \times 10^{-3} \mathrm{~cm}^{-3}$ at $r_{\mathrm{W} / \mathrm{H}}$, the densities of these ions equal $8.8 \times 10^{-11}, 3.4 \times 10^{-9}$, and $1.0 \times 10^{-10} \mathrm{~cm}^{-3}$ respectively, as expected from Figure $2 \mathrm{~b}$. In model $\mathrm{B}$ the column densities of the high-ions are $\sim 10$ times larger, because of the slightly lower bounding pressure and larger ionization parameter at the cloud edge, but more importantly because of the significantly larger cloud size. Negligible amounts of $\mathrm{N} \mathrm{V}$ and O VI are produced in these models.

To order-of-magnitude, the maximal column density of a high-ion may be expressed as

$$
N_{\text {high }} \simeq 2 \max \left[n_{\text {high }}\right] r_{\mathrm{W} / \mathrm{H}}
$$

where $\max \left[n_{\text {high }}\right]$ is the maximum ion density in the cloud, and where we adopt the cloud diameter $2 r_{\mathrm{W} / \mathrm{H}}$ as a characteristic length scale for the WIM. For example, for model A, where $n_{\mathrm{CIV}, \max }=8.8 \times 10^{-11} \mathrm{~cm}^{-3}$ and $r_{\mathrm{W} / \mathrm{H}}=1.3 \mathrm{kpc}$, equation $(10)$ gives a peak $\mathrm{C}$ IV column density of $7.1 \times 10^{11} \mathrm{~cm}^{-2}$, roughly consistent with the maximal column of $2.8 \times 10^{11} \mathrm{~cm}^{-2}$ occurring at a projected radius of $1.1 \mathrm{kpc}$ (see Figure $3 \mathrm{~b}$ ).

For a given bounding pressure, the cloud size $2 r_{W / H}$ is proportional to the $1 / e$ gas scale height for the ionized gas. It therefore follows from equations (8) and (9) that

$$
\frac{N_{\text {high }}}{N_{\text {low }}} \propto \frac{\max \left(n_{\text {high }}\right)}{n_{\mathrm{H}, 0}} .
$$

The density $\max \left(n_{\text {high }}\right)$ is fixed by the external pressure and radiation field. However, the central density $n_{\mathrm{H}, 0}$ depends on the halo potential and becomes small for underconcentrated halos. Thus, $N_{\text {high }} / N_{\text {low }}$ may be used as a dynamical probe of the halo concentration parameter. For example, the C IV/C II column density ratio increases from $1.1 \times 10^{-4}$ to $9.6 \times 10^{-4}$ from model A to $\mathrm{B}$. The increase in the $\mathrm{C}$ IV/C II ratio is primarily due to the increase in cloud size, from model A to B, as the concentration parameter decreases from 32.5 to 8.15.

In Table 5 we list the $\mathrm{H}$ I and metal absorption-line column densities along lines-of-sight that are coincident with the maximal C IV columns in models A and B. Additional output parameters are also listed. It is immediately evident that the computed column densities are 
largely inconsistent with the high-velocity absorbers towards Mrk 509 and PKS 2155-304. The predicted C IV and Si IV columns are several orders of magnitude below the observed values, and the H I and C II columns are too large. The primary difficulty is that in our pressure-confined models the ionization-parameter is too low.

It is clear that to increase the column densities of species such as C IV and Si IV, via photoionization, to observed values exceeding a few $10^{13} \mathrm{~cm}^{-2}$, the bounding pressure must be reduced significantly. However, if this is done for our low-mass CHVC mini-halos, the gas becomes unbound at the larger radii where such species form with greater efficiency ${ }^{9}$. We therefore consider more massive, "dwarf-galaxy" scale halos, in which the clouds are primarily gravitationally confined. In these models the gas remains bound even when the external pressure is reduced to very low values.

In $\S 3$ we found that the optimal pressure for the production of $\mathrm{C}$ IV in the standard metagalactic field is $P / k \approx 2 \mathrm{~cm}^{-3} \mathrm{~K}$, and that for 0.1 solar metallicity the $\mathrm{C}$ IV density then equals $\sim 7 \times 10^{-10} \mathrm{~cm}^{-3}$. For these conditions, a column density of $\sim 5 \times 10^{13} \mathrm{~cm}^{-2}$ requires a path-length of $25 \mathrm{kpc}$. This length scale is much larger than the optical diameters of dwarf galaxies, or of the larger H I halos surrounding them (Mateo 1998; Young et al. 2003). Very tenuous and extended WIM envelopes might exist around the dwarfs, especially if the halos are very underconcentrated. Alternatively, smaller path-lengths are required if the metallicity and photoionization rates are both enhanced by factors of a few. We consider these possibilities below.

We focus on a series of models, labeled $\mathrm{C}$ through $\mathrm{J}$, with input parameters as listed in Table 4. For most of these we adopt median $(\sigma=0)$ Burkert potentials, and assume representative halo masses $M_{\text {vir }}=2 \times 10^{9} M_{\odot}$ with halo scale velocities $v_{s}=30.0 \mathrm{~km} \mathrm{~s}^{-1}$. Two exceptions are model $\mathrm{H}$, which is a $-5 \sigma$ underconcentrated Burkert halo, and model $\mathrm{I}$, in which we adopt an NFW profile. In all models (except J) we choose a total gas mass such that the resulting peak $\mathrm{H}$ I columns are in the range of $1-2 \times 10^{21} \mathrm{~cm}^{-2}$ as observed in the Local Group dwarf galaxies Leo A and Sag DIG (Young \& Lo 1996; 1997). In model $\mathrm{J}$ we reduce the total gas mass such the cloud consists of WIM only, without a neutral core.

The parameters for model C are identical to those adopted by SMW in their dwarf galaxy model, and include a small contribution of stars to the total gravitational potential. In our models we vary the bounding pressures from 1.0 to $0.01 \mathrm{~cm}^{-3} \mathrm{~K}$, consider photoionization by the "standard" or "maximal" radiation fields, and increase the metallicity from 0.1 to 0.3. The resulting photoionization structures are displayed in Figures 5 through 12 . In Table 6

\footnotetext{
${ }^{9}$ This follows directly from the fact that in models A \& B the gas at $r_{\mathrm{W} / \mathrm{H}}$ is just marginally bound for the assumed bounding pressures of 50 and $20 \mathrm{~cm}^{-3} \mathrm{~K}$.
} 
we list output data, including the $\mathrm{H} \mathrm{I}$ and metal column densities along the representative lines-of-sight for which the C IV columns are largest.

In models $\mathrm{C}, \mathrm{D}$ and $\mathrm{E}$, displayed in Figures 5, 6 and 7, we assume photoionization by the standard field, and a metallicity of 0.1 . In this sequence we reduce the bounding pressure from 1.0, to 0.1 to $0.01 \mathrm{~cm}^{-3} \mathrm{~K}$ respectively. In these models most of the gas is WNM, with $M_{\mathrm{WNM}} \cong 1.2 \times 10^{7} M_{\odot}$, and the ionization fronts occur at fixed radii $r_{\mathrm{WNM}}=1.2 \mathrm{kpc}$. The outer edges of the WIM envelopes occur at $r_{\mathrm{W} / \mathrm{H}}$ equal to 4.2, 7.7, and $13.6 \mathrm{kpc}$. Because the gas densities decrease rapidly with radius the WIM masses are essentially independent of the bounding pressures, and $M_{\mathrm{WIM}} \cong 4 \times 10^{6} M_{\odot}$.

A plausible lower-bound to the IGM pressure in the Local Group may be set by adopting the mean cosmic baryon density $n_{b}=2.5 \times 10^{-7} \mathrm{~cm}^{-3}$ as a minimum density and assuming a virial temperature $T \approx 4.3 \times 10^{5} \mathrm{~K}$ corresponding to the Local Group velocity dispersion of $60 \mathrm{~km} \mathrm{~s}^{-1}$ (Sandage 1986). Thus, at large distances from the Galaxy and M31, where the overdensities may be low, IGM pressures as low as $0.2 \mathrm{~cm}^{-3} \mathrm{~K}$ may prevail. Recent Chandra and XMM-Newton X-ray absorption-line observations of highly ionized O VII and O VIII imply pressures in the range $0.5-3.5 \mathrm{~cm}^{-3} \mathrm{~K}$ for hot gas in the Local Group (Nicastro et al. 2002; see also Rasmussen, Kahn \& Paerls 2003). We note further that for $10^{4} \mathrm{~K}$ gas, and a pressure of $0.1 \mathrm{~cm}^{-3} \mathrm{~K}$ the gas recombination time is $\sim 2.6 \times 10^{10} \mathrm{yr}$, so that ionization equilibrium for the high-ions can be reached within a Hubble time at such or larger pressures.

As expected, in the WNM cores of our dwarf galaxy models the column densities of the low-ions are proportional to $N_{\mathrm{HI}}$. The N I and O I densities again fall sharply beyond the ionization fronts, but in contrast to our CHVC models, the outer parts of the WIM envelopes become highly ionized. In particular, in our dwarf galaxy models C II, Si II, and S II, are no longer the dominant ionization stages in the outer parts of the WIM. In models $\mathrm{C}, \mathrm{D}$, and $\mathrm{E}$, the $\mathrm{C}$ IV density reaches maximal values of $7.2 \times 10^{-10}$ at $r \approx 3.2 \mathrm{kpc}$ where $P / k \approx 3.2 \mathrm{~cm}^{-3} \mathrm{~K}$. The peak $\mathrm{C}$ IV column density increases as $P_{\mathrm{HIM}}$ is reduced from 1 to $0.1 \mathrm{~cm}^{-3}$ because as the pressure is reduced the cloud size and projected pathlengths increase. However, reducing the pressure further to $0.01 \mathrm{~cm}^{-3}$ does not lead to a significant increase in the C IV column because of the rapidly declining ion densities at these pressures. In these models, peak C IV columns of $1-2 \times 10^{13} \mathrm{~cm}^{-2}$ occur at projected radii $\sim 2.6 \mathrm{kpc}$ from the cloud centers. The column densities are consistent with equation (10) to order-of-magnitude.

As indicated by Figure 2, O VI is maximized at $P / k=0.1 \mathrm{~cm}^{-3} \mathrm{~K}$, or $n_{\mathrm{H}}=1 \times$ $10^{-5} \mathrm{~cm}^{-3}$. For a metallicity of 0.1 , the optimal O VI density is $1 \times 10^{-10} \mathrm{~cm}^{-3}$. Thus, in models $\mathrm{C}$ and $\mathrm{D}$, the peak $\mathrm{O}$ VI columns increase from $2.9 \times 10^{11}$ to $2.7 \times 10^{12} \mathrm{~cm}^{-2}$ as $P_{\mathrm{HIM}}$ is reduced from 1 to $0.1 \mathrm{~cm}^{-3} \mathrm{~K}$. However, reducing the pressure further as in model $\mathrm{E}$ does 
not lead to a significant increase in the O VI columns.

The predicted C IV and Si IV columns in this first set of dwarf galaxy models are much larger than we found for the CHVCs, but are about a factor of ten smaller than those observed in the C IV HVCs. However, increasing the radiation intensity and the metallicity can bring the predicted columns into harmony with the observations. Thus, in model F we set $P_{\mathrm{HIM}} / k=1 \mathrm{~cm}^{-3}$, and keep the metallicity at 0.1 , but now adopt our maximal radiation field as given by equation (5). As shown by Figure 8, the elevated photoionization rates increase the resulting WIM metal columns by factors of 2-3. In model G we increase the column densities further (see Figure 9) by lowering the bounding pressure to $0.1 \mathrm{~cm}^{-3}$ (thereby increasing the C IV pathlengths), by increasing the metallicity to 0.3 , and again using the maximal field. In model $\mathrm{G}$ the peak C IV column density is $1.5 \times 10^{14} \mathrm{~cm}^{-2}$, comparable to or greater than the observed values in the $\mathrm{C}$ IV absorbers.

With the exception of O VI, model G provides a satisfactory match to the relative and absolute metal column densities in the -283 and $-228 \mathrm{~km} \mathrm{~s}^{-1} \mathrm{C}$ IV absorbers towards Mrk 509, and the $-256 \mathrm{~km} \mathrm{~s}^{-1}$ absorber towards PKS 2155-304. An interesting difficulty is encountered for the $-140 \mathrm{~km} \mathrm{~s}^{-1}$ PKS 2155-304 absorber. As an illustrative example, in Figure 9c we plot the ratios, $N_{\text {model }} / N_{\text {observed }}$, of the columns computed in model G and the observed columns in the $-283 \mathrm{~km} \mathrm{~s}^{-1}$ Mrk 509 absorber (see Tables 2 and 3), as functions of impact parameter in model G. This plot reveals the range of model G sight-lines that are consistent with the observed ion column densities, including the upper and lower-limits. In the shaded area in Figure $9 \mathrm{c}$ the model and measured columns are in agreement to within \pm 0.5 dex. For each of the species, the arrows indicate the range of radii (between the arrowheads) where the model columns are within 0.5 dex of the measured values or limits. A successful fit is achieved if all of the indicated ranges overlap.

Figure 9c shows that lines-of-sight from 0.0 to $3.9 \mathrm{kpc}$ from the cloud center produce an adequate amount of $\mathrm{C}$ IV. No constraints are provided by $\mathrm{N}$ V and S III, for which the computed values are consistent with the observed upper-limits for all lines-of-sight. The N I, Si II, and S II upper-limits are consistent with impact parameters of at least 1.2, 1.8, and $1.0 \mathrm{kpc}$ respectively. A strong constraint is provided by $\mathrm{C} \mathrm{II}$, which restricts the projected radii to the range $2-2.6 \mathrm{kpc}$. The measured column densities of all other species, with the exception of O VI, are consistent with the model for $2-2.6 \mathrm{kpc}$. The observed O VI column cannot be reproduced for any line-of-sight. We note that the line-of-sight for which $\mathrm{C}$ IV is maximized occurs at $2.3 \mathrm{kpc}$, within the range constrained by $\mathrm{C}$ II. For this radius, the predicted C IV, Si III, and Si IV columns are equal to $1.5 \times 10^{14}, 3.3 \times 10^{13}$, and $2.0 \times 10^{13} \mathrm{~cm}^{-2}$, respectively, compared to the observed columns, of $\sim 1.5 \times 10^{14} \mathrm{~cm}^{-2}$ for C IV $\sim 2 \times 10^{13} \mathrm{~cm}^{-2}$ for Si III, and $\sim 3 \times 10^{13} \mathrm{~cm}^{-2}$ for Si IV. At $2.3 \mathrm{kpc}$ the predicted 
$\mathrm{H}$ I and C II columns of $1.8 \times 10^{16}$ and $6.5 \times 10^{13} \mathrm{~cm}^{-2}$ are consistent with the observed H I upper-limit and measured C II column of $4.3 \times 10^{-2} \mathrm{~cm}^{-2}$. As already indicated, at this radius, the remaining computed columns are consistent with all of the upper-limits. Of specific interest is $\mathrm{N} \mathrm{V}$, for which our predicted column of $7.1 \times 10^{12} \mathrm{~cm}^{-2}$ is close to the observed upper-limit of $1.5 \times 10^{13} \mathrm{~cm}^{-2}$.

Similar fits may be found for the $-228 \mathrm{~km} \mathrm{~s}^{-1}$ Mrk 509 and $-256 \mathrm{~km} \mathrm{~s}^{-1}$ PKS 2155304 absorbers. The range of radii in model $\mathrm{G}$ that are consistent with the data (excepting $\mathrm{O} \mathrm{VI}$ ) in these absorbers are 3.1 to $3.4 \mathrm{kpc}$ and 3.2 to $3.5 \mathrm{kpc}$, respectively. However, the $-140 \mathrm{~km} \mathrm{~s}^{-1}$ PKS 2155-304 absorber appears anomalous in that the abundance ratio of low-ions to high-ions is larger in this system compared to the other absorbers. In particular, sight lines with impact parameter between 3.1 to $5.0 \mathrm{kpc}$ consistent with the C IV columns are underabundant in the low-ions C II, Si II and Si III and also H I, while more centrally confined sight lines, from 2.1 to $2.5 \mathrm{kpc}$ are consistent with these low ions, but yield an excess of C IV.

We conclude that photoionized WIM envelopes around dwarf galaxies are natural sites for the production of C IV, Si III, and Si IV ions, and the simultaneous reduction of lowions such as C II, Si II, and S II. Ion column densities ranging from $10^{13}$ to $10^{14} \mathrm{~cm}^{-2}$, comparable to those in the C IV HVCs, are possible, provided the IGM pressure is low with $P / k \lesssim 1 \mathrm{~cm}^{-3} \mathrm{~K}$ as found by Sembach et al. (1999). If median $\Lambda$ CDM Burkert halos are good representations of dwarf galaxy dark-matter halos, the implied gas path-lengths require metallicities $\gtrsim 0.3$ to produce such large metal columns in individual dwarf galaxies. However, photoionization by metagalactic radiation is not an efficient source of $\mathrm{N}$ V or $\mathrm{O}$ VI in such WIM envelopes.

Model $\mathrm{H}$ is a $-5 \sigma$ Burkert halo, and illustrates the effect of reducing the halo concentration parameter. In making this reduction we keep $v_{s}$ fixed at $30.0 \mathrm{~km} \mathrm{~s}^{-1}$. The associated halo mass is then $M_{\text {vir }}=6.2 \times 10^{9} M_{\odot}$. In Figure 10 we display results for the standard field, $P_{\mathrm{HIM}} / k=1 \mathrm{~cm}^{-3} \mathrm{~K}$, and a metallicity of 0.1 . We choose a gas mass $M_{\mathrm{WM}}=1.3 \times 10^{9}$ that yields a peak H I column of $1.4 \times 10^{21} \mathrm{~cm}^{-2}$. The column densities of the low-ions in the WNM core are therefore unchanged with respect to the corresponding median halo model $\mathrm{C}$. However, the cloud size in model $\mathrm{H}$ is significantly larger with $r_{\mathrm{W} / \mathrm{H}}=25 \mathrm{kpc}$. The column densities of the high-ions are therefore enhanced. For example the peak $\mathrm{C}$ IV column for this model is $7.5 \times 10^{13} \mathrm{~cm}^{-2}$. In moving from model $\mathrm{C}$ to $\mathrm{H}$ the $\mathrm{C}$ IV/C II column density ratio increases from $2.4 \times 10^{-4}$ to $1.3 \times 10^{-3}$, consistent with equation (11).

In model I, displayed in Figure 11, we consider a dwarf galaxy model using a NFW profile. We adopt a $-4 \sigma$ underconcentrated halo to yield a neutral gas scale height similar to those in our median Burkert models which match the observed HI gas distributions in Leo A 
and Sag DIG (SMW). We again fix $v_{s}=30 \mathrm{~km} \mathrm{~s}^{-1}$. The virial mass is then $4.9 \times 10^{9} M_{\odot}$. For a direct comparison with model $\mathrm{G}$ we show results for the maximal field, $P_{\mathrm{HIM}}=0.1 \mathrm{~cm}^{-3} \mathrm{~K}$, and a metallicity of 0.3 . A total gas mass of $3.1 \times 10^{7} M_{\odot}$ is required to produce an $\mathrm{H} \mathrm{I} \mathrm{column}$ of $1.4 \times 10^{21} \mathrm{~cm}^{-2}$. The projected neutral gas $1 /$ e gas scale height is $0.4 \mathrm{kpc}$. In our NFW model the cloud radius $r_{\mathrm{W} / \mathrm{H}}=12.4 \mathrm{kpc}$ (as opposed to $7.4 \mathrm{kpc}$ in model G). The larger size results in part from the fact that the gas distributions in NFW potentials are exponentials and decrease less rapidly than in Burkert potentials where the gas distributions are Gaussians (SMW). The larger cloud radius leads to correspondingly larger column densities for the high-ions. The peak C IV column in this model is $2.6 \times 10^{14} \mathrm{~cm}^{-2}$.

In the dwarf galaxy models we have discussed so far, the WIM components exist as envelopes around the WNM cores. Furthermore, the large opacities associated with the peak $\mathrm{H}$ I columns of $1.4 \times 10^{21} \mathrm{~cm}^{-2}$ in these models imply that the external metagalactic field cannot alone provide the gas heating for the WNM. As found by SMW, for median Burkert halos with $M_{\mathrm{vir}}=2 \times 10^{9} M_{\odot}$ a maximum gas mass of $3 \times 10^{6} M_{\odot}$ can be maintained at $\sim 10^{4} \mathrm{~K}$ by the present-day metagalactic field. Internal heat sources, such as stars, are required to maintain larger WNM masses in dwarf galaxies. However, the properties of the WIM envelopes, which may be fully ionized and heated by the external field, do not depend significantly on the presence of the WNM cores. In particular, "fully ionized dwarf galaxies" could exist, in which the dark-matter halos never contained sufficient gas mass for the formation of neutral cores and stars, but which contain photoionized gas detectable via UV metal absorption lines.

To demonstrate this, we conclude with model $\mathrm{J}$, which represents such a fully ionized dwarf galaxy. The parameters are identical to model $\mathrm{G}$, except that we reduce the gas mass to $M_{\mathrm{WM}}=1 \times 10^{6} \mathrm{~cm}^{-2}$. The resulting peak H I column density is now only $5 \times 10^{17} \mathrm{~cm}^{-2}$, so the cloud remains optically thin and fully ionized. The gas cloud is entirely WIM. The cloud radius $r_{\mathrm{W} / \mathrm{H}}=4.7 \mathrm{kpc}$ is smaller than in model $\mathrm{G}$ because the WNM core has been removed. As is indicated by Figure 12 and Table 6 the resulting metal columns are reduced, but only by about $30 \%$.

\section{Discussion and Summary}

In this paper we study the distributions and relative abundances of metal ions in gas clouds that are confined in low-mass dark-matter mini-halos in the extended Galactic halo or Local Group, and which are photoionized by the present-day metagalactic radiation field. We consider spherical and hydrostatic clouds, with gas temperatures $\sim 10^{4} \mathrm{~K}$, which are also subjected to external bounding pressures provided by a hot medium. We compute the 
distributions of low-ions, C II, N I, O I, Si II, and S II, produced primarily in the optically thick WNM cores, and high-ions, C IV, N V, O VI, Si III, Si IV, and S III, produced in the outer WIM shielding envelopes of the clouds. We study the ionization properties of the compact H I high-velocity clouds, as photoionized pressure-confined gas clouds in $\sim 10^{8} \mathrm{M}_{\odot}$ mini-halos. We then focus on the metal ion distributions in more massive $\sim 2 \times 10^{9} \mathrm{M}_{\odot}$ halos at the dwarf-galaxy scale. In particular, we determine conditions for the formation of highly-ionized components, with ionization states similar to the high-velocity C IV absorbers observed by Sembach et al. (1999) and Collins et al. (2004) towards Mrk 509 and PKS 2155304.

First, we address the question of whether the C IV HVCs could be the ionized counterparts or envelopes of pressure-confined $\mathrm{H}$ I CHVCs, which are photoionized by metagalactic radiation, where external bounding pressures $P / k \sim 50 \mathrm{~cm}^{-3} \mathrm{~K}$ are required to bind, compress, and neutralize the $\mathrm{H}$ I clouds. We find that in such objects the ionization parameter is too low to allow efficient production of C IV and other high-ions. The dominant ionization stages in these objects remain C II, N I, O I, Si II, and S II. If the CHVCs are pressureconfined circumgalactic objects (as favored by SMW) then significant columns of high-ions require alternative ionization mechanisms, as suggested by Fox et al. (2003) for the relatively nearby Complex C. One attractive possibility to be considered is the role of non-equilibrium ionization in the conductive interfaces that must exist between the WIM envelopes of minihalo clouds and surrounding hot gas. Our computations, and the recent O VI detections, confirm the conclusions of Sembach et al. (2000; 2003) that additional processes are likely affecting the WIM ionization properties in the Mrk 509 and PKS 2155-304 absorbers. In the WNM cores, the column densities of the low-ions and atoms are predicted to be equal to the metal abundance times the H I column along the line-of-sight. UV absorption-line observations of such low-ions can therefore provide useful and direct measures of the CHVC metallicities, if photoionization dominates in the cores.

Second, we study the properties of more extended and tenuous photoionized WIM envelopes around more massive, dwarf galaxy scale, mini-halos. In such systems, gravitational confinement of the gas is more effective, and the bounding pressures can be taken to much lower values. The ionization parameters in the WIM may therefore be increased to values that yield large abundances of C IV, S III, Si IV, and Si III, and simultaneously, reduced abundances of low-ions. We find that ionization states comparable to those in the C IV HVCs are produced in the WIM envelopes of dwarf galaxies for bounding pressures $P / k \lesssim 1 \mathrm{~cm}^{-3} \mathrm{~K}$. An important exception is O VI, which is inefficiently produced by photoionization.

In our models, the predicted metal column densities are proportional to the cloud sizes and associated path-lengths. For a given gas velocity dispersion, the cloud size-scale is fixed 
by the dark-matter gravitational potential. For $\Lambda$ CDM Burkert halos with median darkmatter concentration parameters, we find that the WIM envelopes extend out to 5-10 kpc from the cloud centers, depending on the value of the bounding pressure. C IV columns of up to $\sim 10^{14} \mathrm{~cm}^{-2}$, as observed by Sembach et al. (1999), would then require metallicities of at least 0.3 times solar for absorption occurring in a single WIM envelope. We conclude that the WIM envelopes of dwarf galaxies are natural sites for the formation of C IV, S III, Si IV, and Si III via metagalactic photoionization provided $P / k \lesssim 1 \mathrm{~cm}^{-3} \mathrm{~K}$ in the surrounding IGM. This is consistent with the finding of Sembach et al. (1999) that such low pressures favor the production of C IV. Such low pressures appear to be indicated by recent X-ray absorption-line observations of very highly-ionized O VII and O VIII that may be probing the hot IGM medium in the Local Group (Nicastro et al. 2002; Rasmussen, Kahn \& Paerels 2003).

We also present results for fully ionized dwarf galaxies, in which the gas mass confined to the mini-halos remains optically thin. In such objects WNM cores do not exist and the H I column densities are correspondingly low. Such objects are also expected to be starless given that photoionization was more effective at earlier epochs, at least since reionization (Efstathiou 1992; Kepner, Babul \& Spergel 1997; Barkana \& Loeb 1999). Our computations show that such "dark galaxies" could be detectable as UV metal-line absorbers, with ionization states similar to those observed in the C IV absorbers. However, given the relatively high metallicities required, the gas that was trapped in such halos must have been previously enriched.

The origin, nature, and location of the C IV HVCs remain uncertain. Sembach et al. (1999) suggested that the absorbers towards Mrk 509 and PKS 2155-304 may be related to the group of H I HVCs observed in the "Galactic center negative" (GCN) direction (Wakker \& van Woerden 1991). Some of these GCN clouds have velocities close to the C IV HVCs, and are located within $5^{\circ}$ of them. The GCN clouds may be tracing a system of lowsurface brightness galaxies (Mirabel \& Cohen 1979), although an origin in the Magellanic Stream has also been suggested (Wakker \& van Woerden 1991). Our models suggest that a system of ionized dwarf galaxies, perhaps associated with the GCN H I clouds viewed as low-surface brightness galaxies, could, with the exception of O VI, give rise to UV metal absorption features similar to those observed in the C IV HVCs.

\section{Acknowledgments}

We thank the US-Israel Binational Science Foundation (grant 2002317) for supporting our research. We thank C.F. McKee for discussions, and the anonymous referee for helpful 
advice and suggestions. We thank Gary Ferland for maintaining CLOUDY as a publicly available code.

\section{REFERENCES}

Barkana, R. \& Loeb, A. 1999, ApJ, 523, 54

Bernstein, R. A., Freedman, W. L., \& Madore, B. F. 2002, ApJ, 571, 56

Blitz, L., Spergel, D. N., Teuben, P. J., Hartmann, D., \& Burton, W. B. 1999, ApJ, 514, 818

Boksenberg, A., Sargent, W. L. W., \& Rauch, M. 2003, ApJS, submitted, astro-ph/0307557

Burkert, A. 1995, ApJ, 447, L25

Braun, R. \& Burton, W. B. 1999, A\&A, 341, 437

Bullock, J. S., Kolatt, T. S., Sigad, Y., Somerville, R. S., Kravtsov, A. V., Klypin, A. A., Primack, J. R., \& Dekel, A. 2001, MNRAS, 321, 559

Burton, W. B., Braun, R., \& Chengalur, J. N. 2001, A\&A, 369, 616

Chen, L.-W., Fabian, A. C., \& Gendreau, K. C. 1997, MNRAS, 285, 449

Collins, J. A., Shull, J. M., \& Giroux, M. L. 2003, ApJ, in press, astro-ph/0312105

Draine, B. T. 2003, ARAA, 41, 241

de Heij, V., Braun, R., \& Burton, W. B. 2002, A\&A, 392, 417

Efstathiou, G. 1992, MNRAS, 256, 43P

Ferland, G. J., Korista, K. T., Verner, D. A., Ferguson, J. W., Kingdon, J. B., \& Verner, E. M. 1998, PASP, 110, 761

Fox, A. J., Savage, B. D., Wakker, B. P., Richter, P., Sembach, K. R., \& Tripp, T. M. 2003, ApJ, in press, astro-ph/0310769

Haardt, F. \& Madau, P. 1996, ApJ, 461, 20

Heckman, T. M., Norman, C. A., Strickland, D. K., \& Sembach, K. R. 2002, ApJ, 577, 691

Holweger, H. 2001, AIP Conf. Proc. 598: Joint SOHO/ACE workshop "Solar and Galactic Composition", 23, ed. R. F. Wimmer-Schweingrube (AIP), 515 
Kepner, J. V., Babul, A., \& Spergel, D. N. 1997, ApJ, 487, 61

Kepner, J., Tripp, T. M., Abel, T., \& Spergel, D. 1999, AJ, 117, 2063

Klypin, A., Kravtsov, A. V., Valenzuela, O., \& Prada, F. 1999, ApJ, 522, 82

Lu, L., Sargent, W. L. W., Barlow, T. A., Churchill, C. W., \& Vogt, S. S. 1996, ApJS, 107, 475

Maller, A. H., Prochaska, J. X., Somerville, R. S., \& Primack, J. R. 2003, MNRAS, 343, 268

Martin, C., Hurwitz, M., \& Bowyer, S. 1991, ApJ, 379, 549

Mateo, M. L. 1998, ARA\&A, 36, 435

Mirabel, I. F. \& Cohen, R. J. 1979, MNRAS, 188, 219

Moore, B., Ghigna, S., Governato, F., Lake, G., Quinn, T., Stadel, J., \& Tozzi, P. 1999, ApJ, 524, L19

Muller, C. A., Oort, J. H., \& Raimond, E. 1963, CR Acad. Sci. Paris, 257, 1661

Murphy, E. M. et al. 2000, ApJ, 538, L35

Navarro, J. F., Frenk, C. S., \& White, S. D. M. 1995, MNRAS, 275, 720

Nicastro, F. et al. 2002, ApJ, 573, 157

Oort, J. H. 1970, A\&A, 7, 381

Prochaska, J. X. \& Wolfe, A. M. 1997, ApJ, 474, 140

Putman, M. E. et al. 2002, AJ, 123, 873

Rasmussen, A., Kahn, S. M., \& Paerels, F. 2003, to appear in "The IGM/Galaxy Connection: The Distribution of Baryons at $\mathrm{z}=0$ " (Kluwer Academic Publishing), astroph/0301183

Sandage, A. 1986, ApJ, 307, 1

Sembach, K. R., Savage, B. D., Lu, L., \& Murphy, E. M. 1999, ApJ, 515, 108

Sembach, K. R. et al. 2000, ApJL, 538, L31

Sembach, K. R., Gibson, B. K., Fenner, Y., \& Putman, M. E. 2002, ApJ, 572, 178 
Sembach, K. R. et al. 2003, ApJS, 146, 165

Shull, J. M., Roberts, D., Giroux, M. L., Penton, S. V., \& Fardal, M. A. 1999, AJ, 118, 1450

Spergel, D. N. et al. 2003, ApJS, 148, 175

Sternberg, A., McKee, C. F., \& Wolfire, M. G. 2002, ApJS, 143, 419

Vogel, S. N., Weymann, R., Rauch, M., \& Hamilton, T. 1995, ApJ, 441, 162

Wakker, B. P. \& van Woerden, H. 1991, A\&A, 250, 509

Wakker, B. P. \& van Woerden, H. 1997, ARA\&A, 35, 217

Wakker, B. P. et al. 2003, ApJS, 146, 1

Wechsler, R. H., Bullock, J. S., Primack, J. R., Kravtsov, A. V., \& Dekel, A. 2002, ApJ, 568,52

Weiner, B. J., Vogel, S. N., \& Williams, T. B. 2001, in ASP Conf. Ser. 240, Gas and Galaxy Evolution, ed. J. E. Hibbard, M. P. Rupen, \& J. H. van Gorkom (San Francisco: ASP), 515

Young, L. M. \& Lo, K. Y. 1996, ApJ, 462, 203

Young, L. M. \& Lo, K. Y. 1997, ApJ, 490, 710

Young, L. M., van Zee, L., Lo, K. Y., Dohm-Palmer, R. C., \& Beierle, M. E. 2003, ApJ, 592, 111 


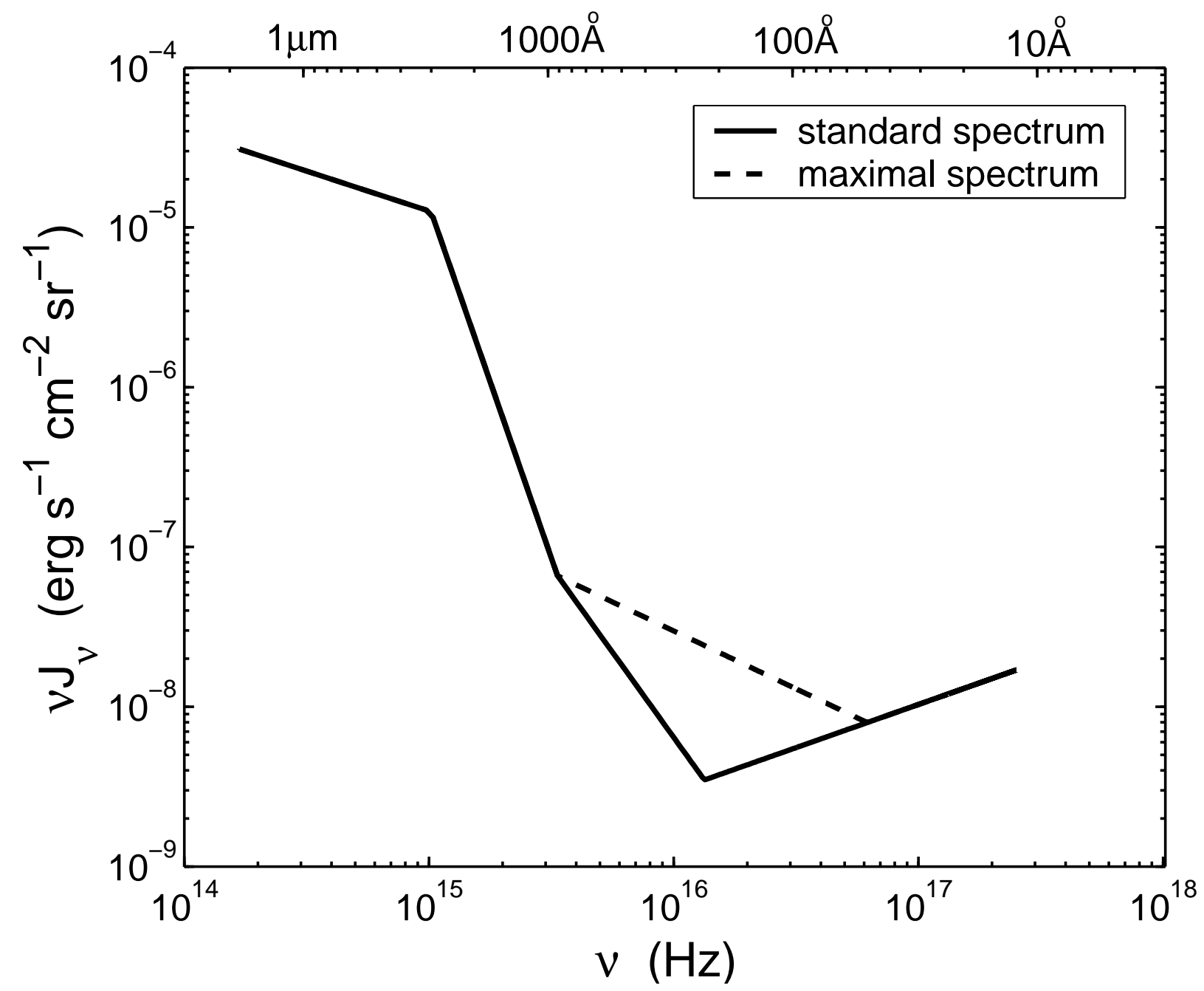

Fig. 1. - The standard metagalactic radiation field (solid line) and the maximal field (dashed line). 


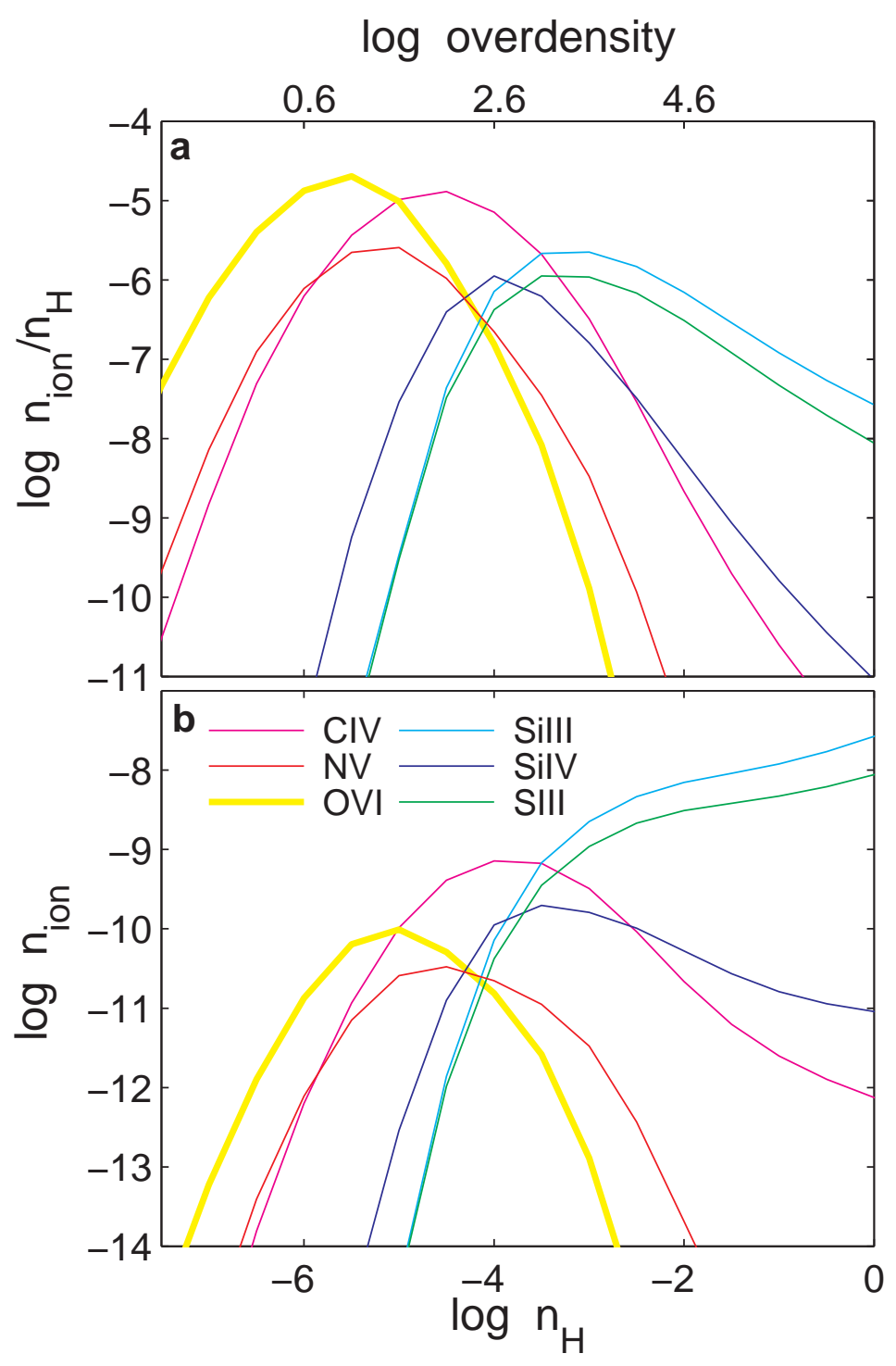

Fig. 2.- The abundances of high-ions as a function of the hydrogen density, for photoionization of $10^{4} \mathrm{~K}$ gas by the standard metagalactic field: panel (a) shows the abundances relative to hydrogen, and panel (b) shows the absolute abundances. Color version is available in the electronic on-line edition. 

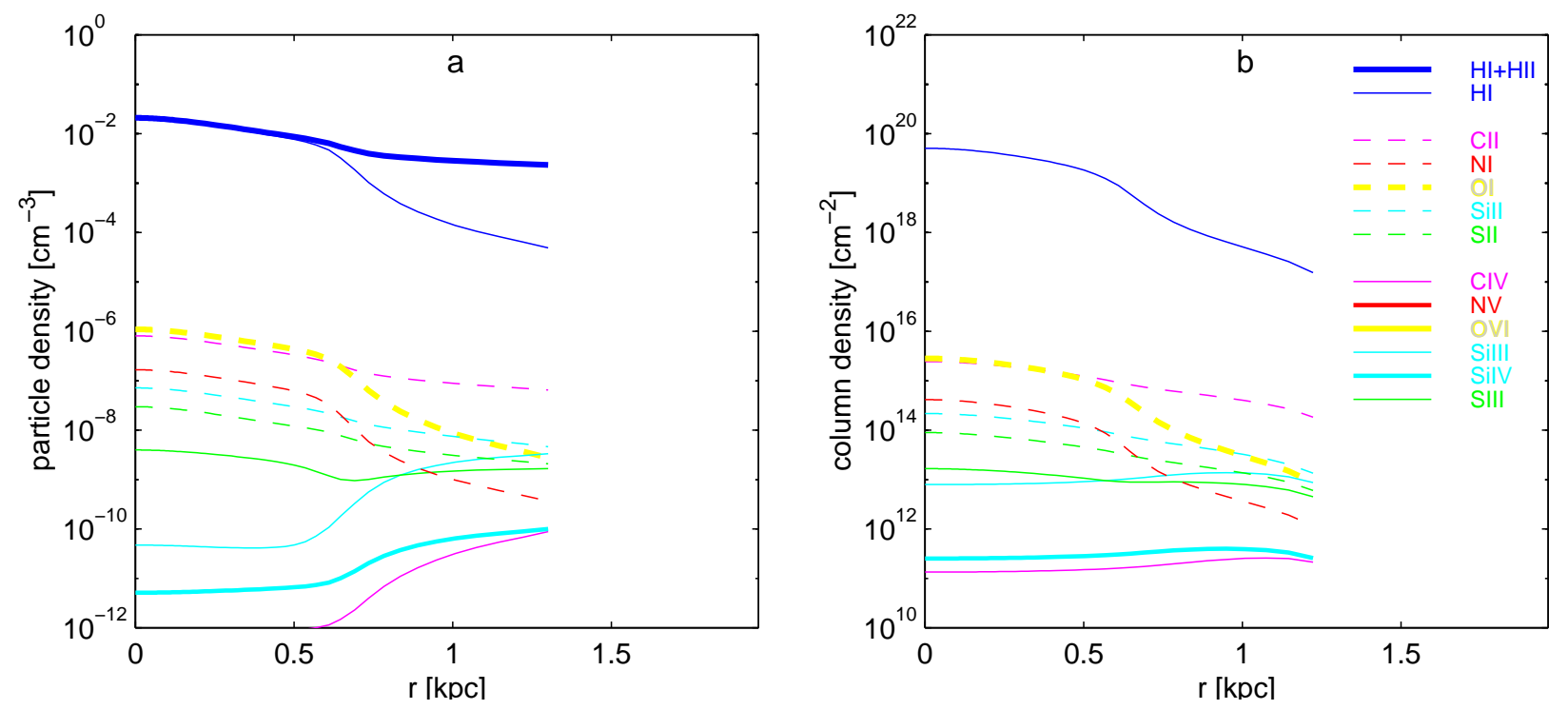

Fig. 3.- Model A: $M_{v i r}=1 \times 10^{8} \mathrm{M}_{\odot}$ median halo, $M_{\text {gas }}=1.1 \times 10^{6} \mathrm{M}_{\odot}, 0.1$ solar metallicity, photoionization by the standard metagalactic field, bounding pressure $P_{\mathrm{HIM}} / k_{B}=$ $50 \mathrm{~cm}^{-3} \mathrm{~K}$. Panel (a) shows the volume densities as a function of the distance from the cloud center, and panel (b) shows the projected column densities
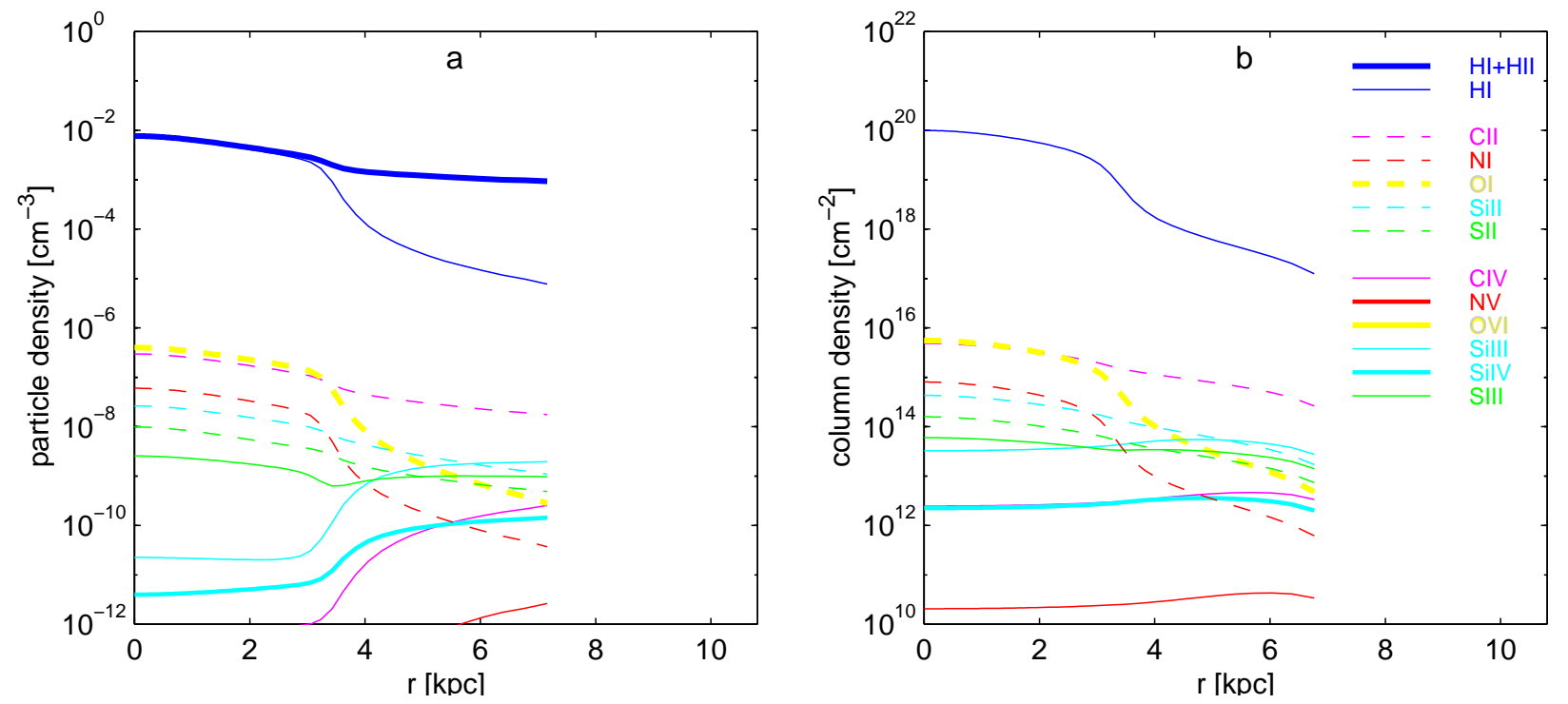

Fig. 4. - Model B: $M_{\text {vir }}=3.2 \times 10^{8} \mathrm{M}_{\odot}-4 \sigma$ halo, $M_{\text {gas }}=7.1 \times 10^{7} \mathrm{M}_{\odot}, 0.1$ solar metallicity, standard radiation field, $P_{\mathrm{HIM}} / k_{B}=20 \mathrm{~cm}^{-3} \mathrm{~K}$. 

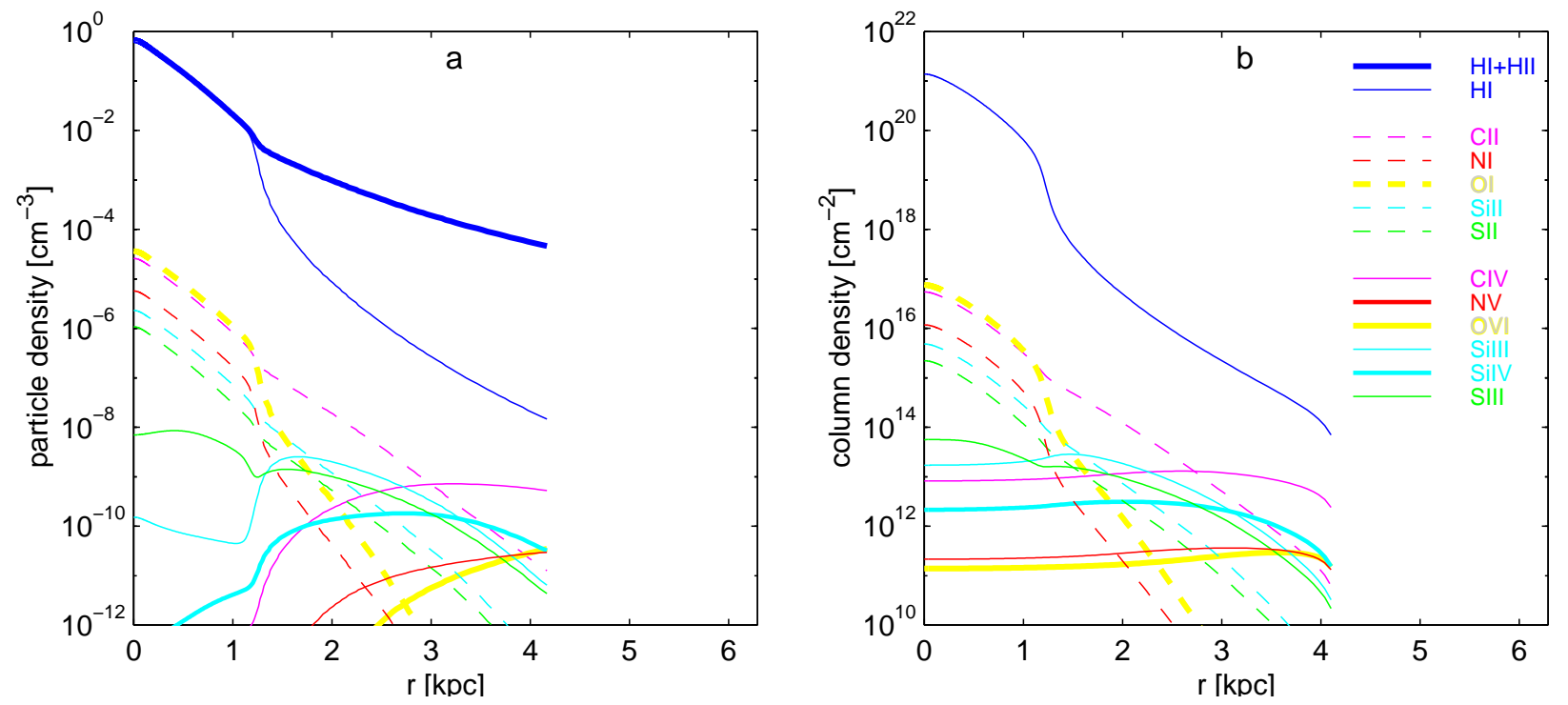

Fig. 5.- Model C: $M_{\text {vir }}=2 \times 10^{9} \mathrm{M}_{\odot}$ median halo, $M_{\text {gas }}=1.6 \times 10^{7} \mathrm{M}_{\odot}$, 0.1 solar metallicity, standard radiation field, $P_{\mathrm{HIM}} / k_{B}=1 \mathrm{~cm}^{-3} \mathrm{~K}$.
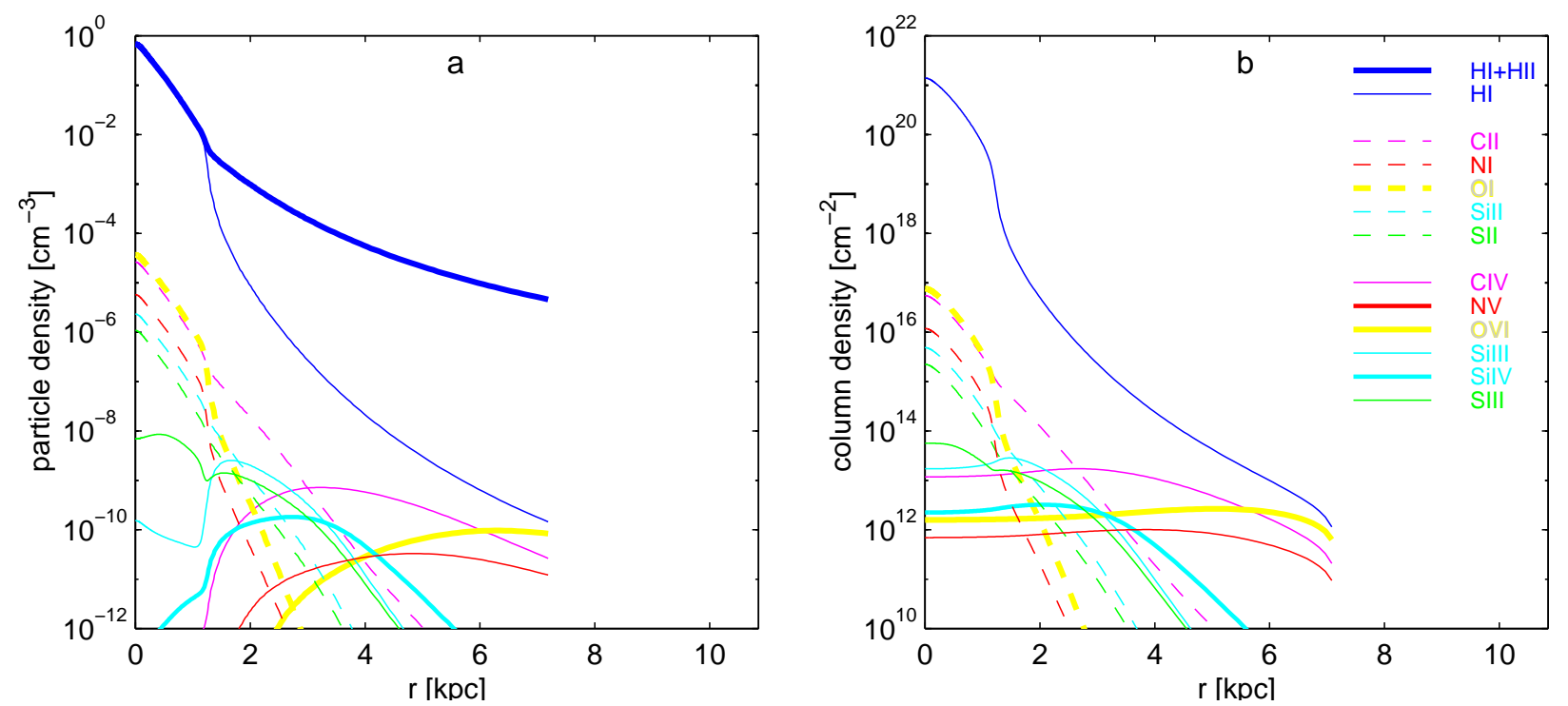

Fig. 6. - Model D: $M_{v i r}=2 \times 10^{9} \mathrm{M}_{\odot}$ median halo, $M_{\text {gas }}=1.6 \times 10^{7} \mathrm{M}_{\odot}, 0.1$ solar metallicity, standard radiation field, $P_{\mathrm{HIM}} / k_{B}=0.1 \mathrm{~cm}^{-3} \mathrm{~K}$. 

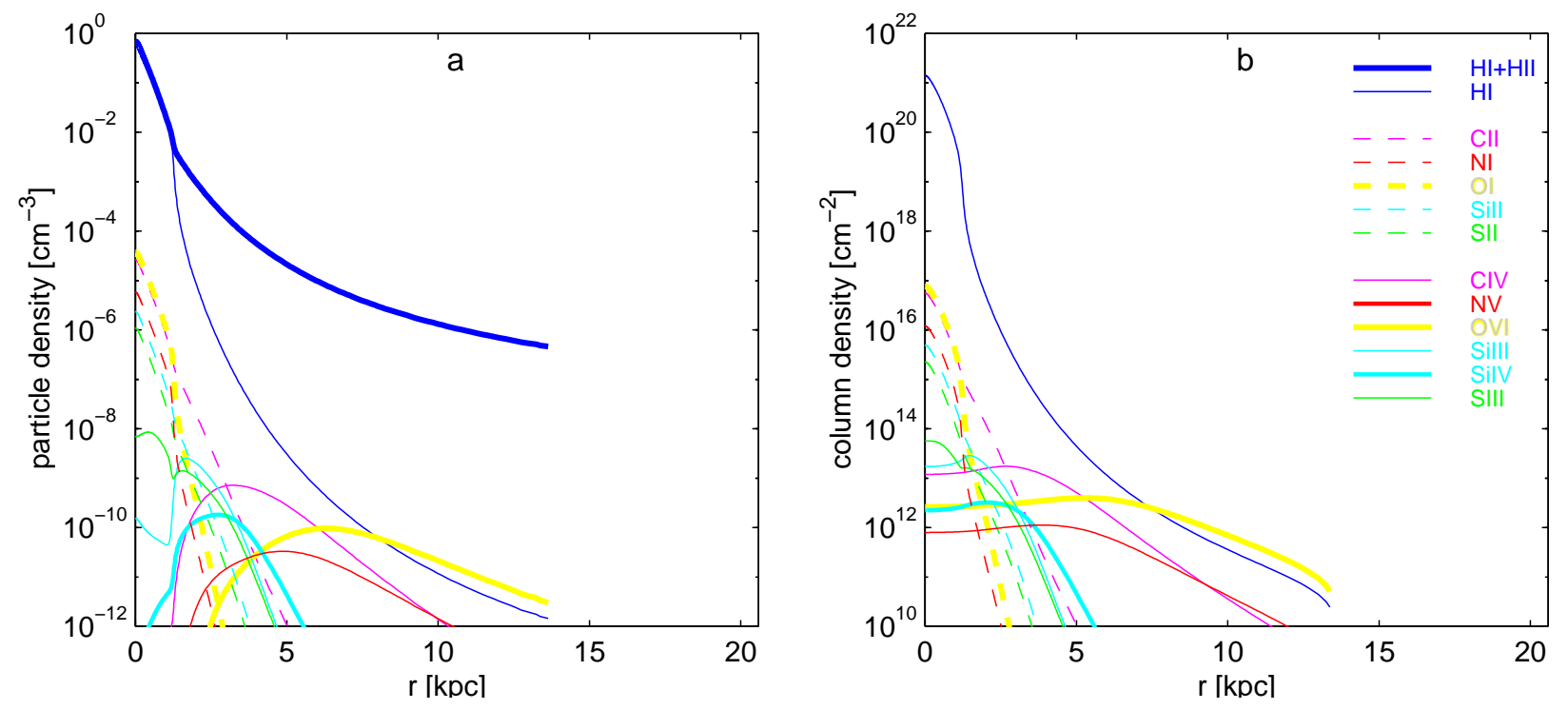

Fig. 7.- Model E: $M_{v i r}=2 \times 10^{9} \mathrm{M}_{\odot}$ median halo, $M_{\text {gas }}=1.7 \times 10^{7} \mathrm{M}_{\odot}$, 0.1 solar metallicity, standard radiation field, $P_{\mathrm{HIM}} / k_{B}=0.01 \mathrm{~cm}^{-3} \mathrm{~K}$.
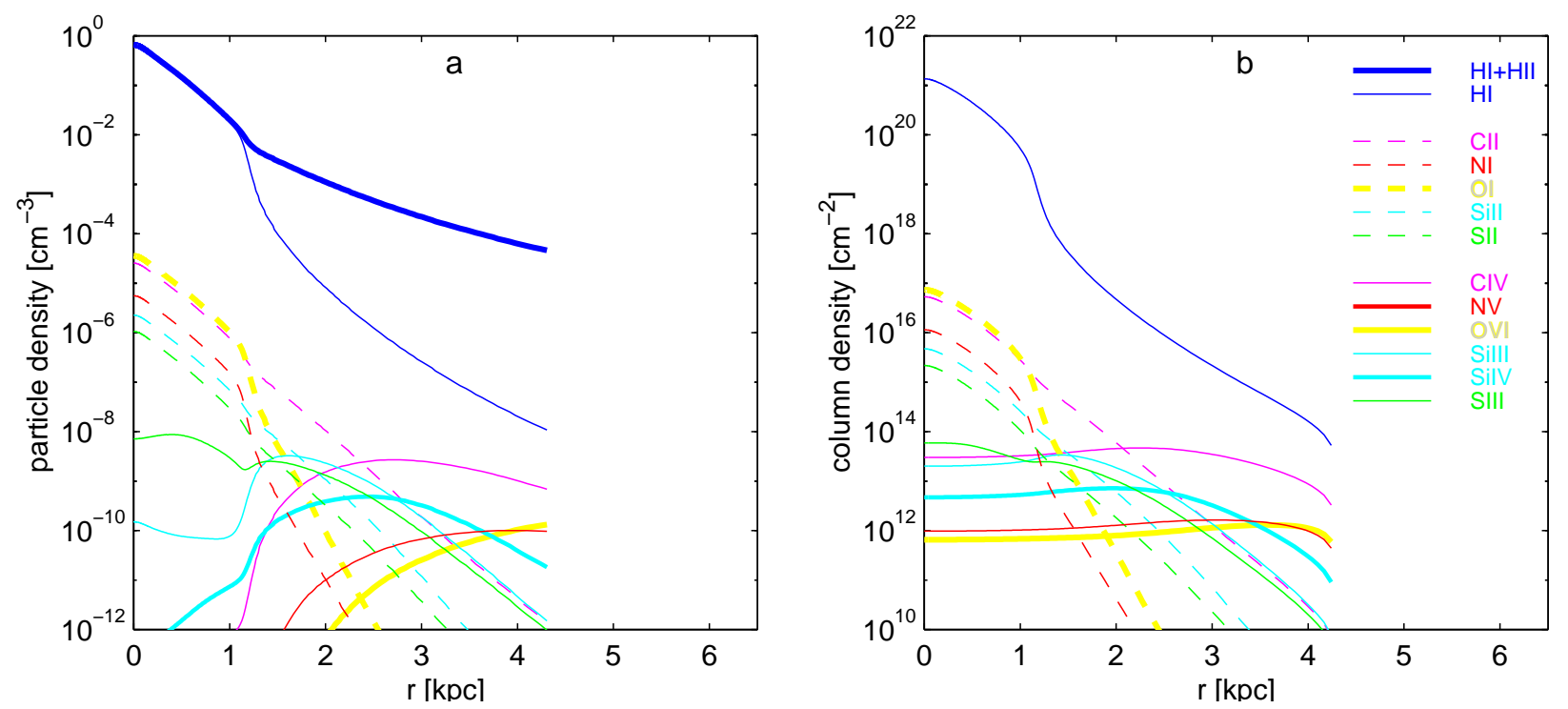

Fig. 8.- Model F: $M_{v i r}=2 \times 10^{9} \mathrm{M}_{\odot}$ median halo, $M_{\text {gas }}=1.6 \times 10^{7} \mathrm{M}_{\odot}, 0.1$ solar metallicity, maximal radiation field, $P_{\mathrm{HIM}} / k_{B}=1 \mathrm{~cm}^{-3} \mathrm{~K}$. 


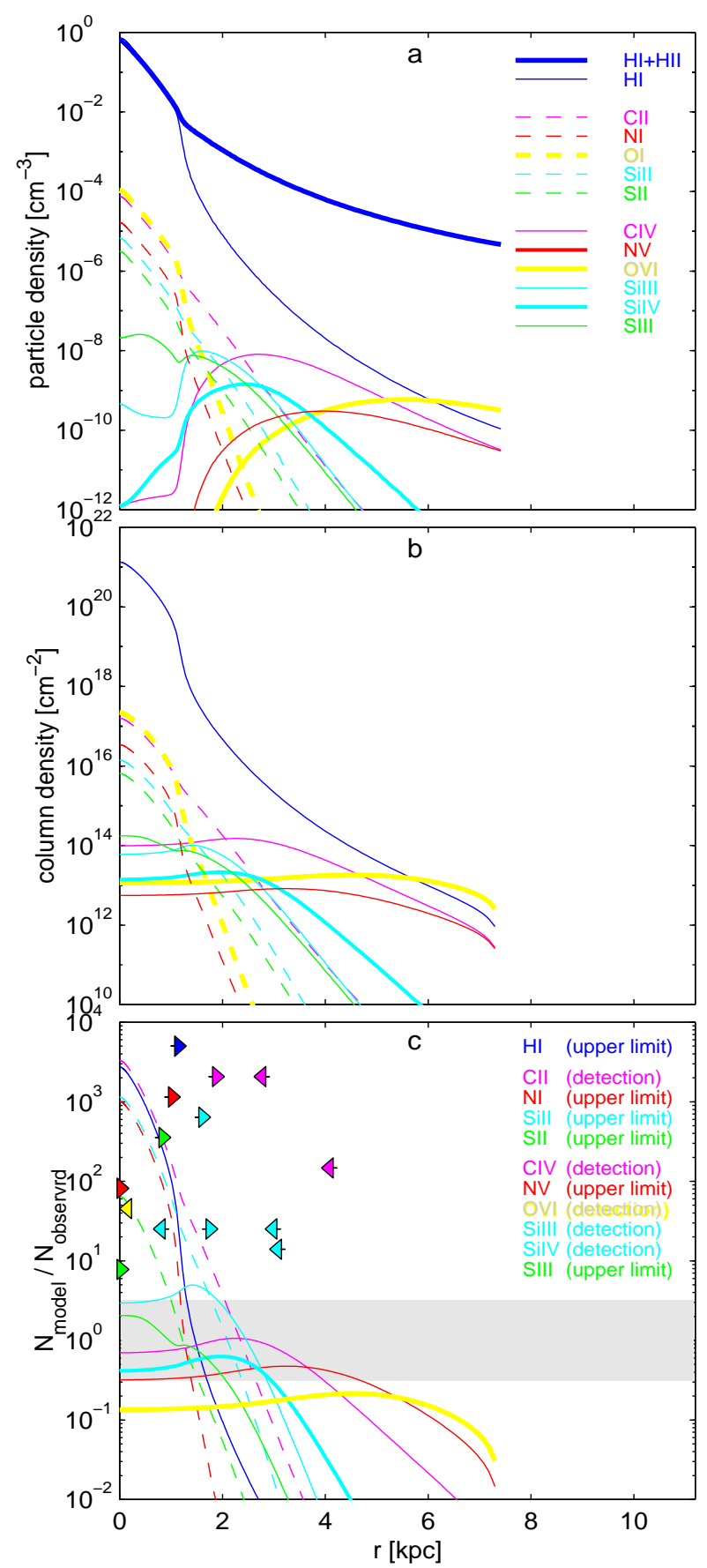

Fig. 9.- Model G: $M_{v i r}=2 \times 10^{9} \mathrm{M}_{\odot}$ median halo, $M_{\text {gas }}=1.6 \times 10^{7} \mathrm{M}_{\odot}, 0.3$ solar metallicity, maximal radiation field, $P_{\mathrm{HIM}} / k_{B}=0.1 \mathrm{~cm}^{-3} \mathrm{~K}$. As before, panel (a) shows the volume densities as a function of the distance from the cloud center, and panel (b) shows the projected column densities. Panel (c) displays, for each ion, the ratio of the calculated model-G columns to the observed columns of the $-300 \mathrm{~km} \mathrm{~s}^{-1}$ component towards Mrk 509 (Collins et al. 2004; see Table 3). The legend indicates species with measured columns, upper limits or lower limits. In the shaded area the calculated and observed values agree to within 0.5 dex. For each of the species, the arrows indicate the range of radii (between the arrowheads) where the model columns are within 0.5 dex of the measured values or limits. 

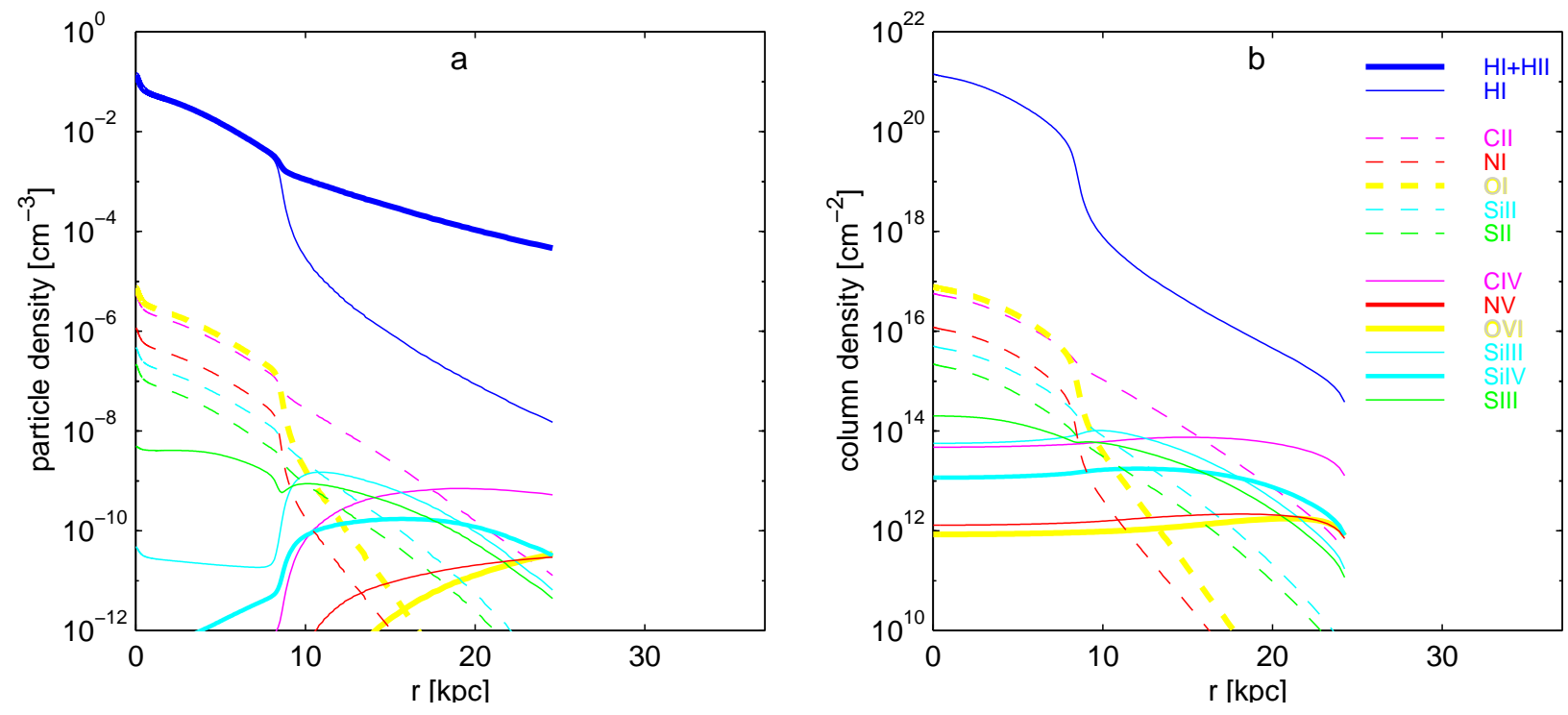

Fig. 10.- Model H: $M_{\text {vir }}=6.2 \times 10^{9} \mathrm{M}_{\odot}-5 \sigma$ halo, $M_{\text {gas }}=1.3 \times 10^{9} \mathrm{M}_{\odot}$, 0.1 solar metallicity, standard radiation field, $P_{\mathrm{HIM}} / k_{B}=1 \mathrm{~cm}^{-3} \mathrm{~K}$.
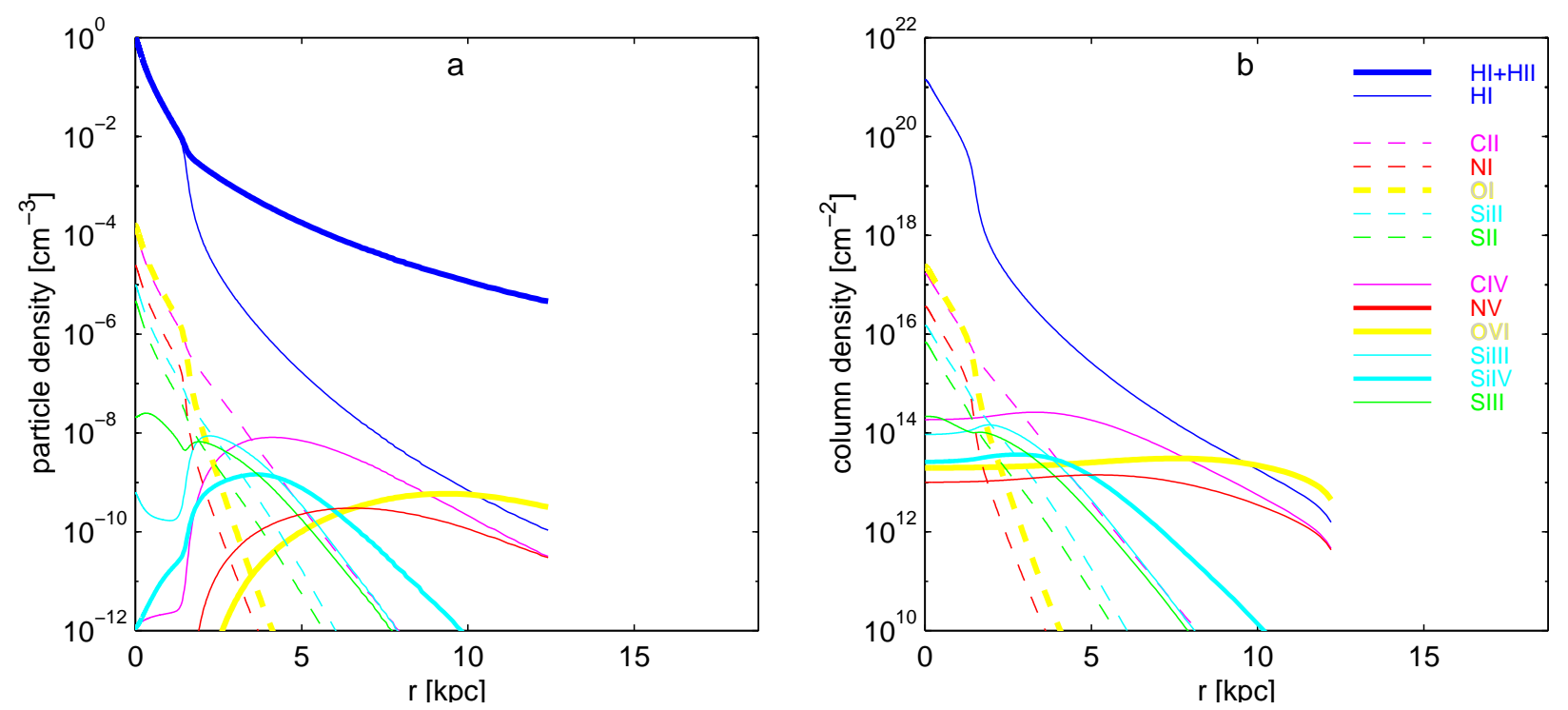

Fig. 11. - Model I: $M_{v i r}=4.9 \times 10^{9} \mathrm{M}_{\odot}-4 \sigma \mathrm{NFW}$ halo, $M_{\text {gas }}=3.1 \times 10^{7} \mathrm{M}_{\odot}, 0.3$ solar metallicity, maximal radiation field, $P_{\mathrm{HIM}} / k_{B}=0.1 \mathrm{~cm}^{-3} \mathrm{~K}$. 

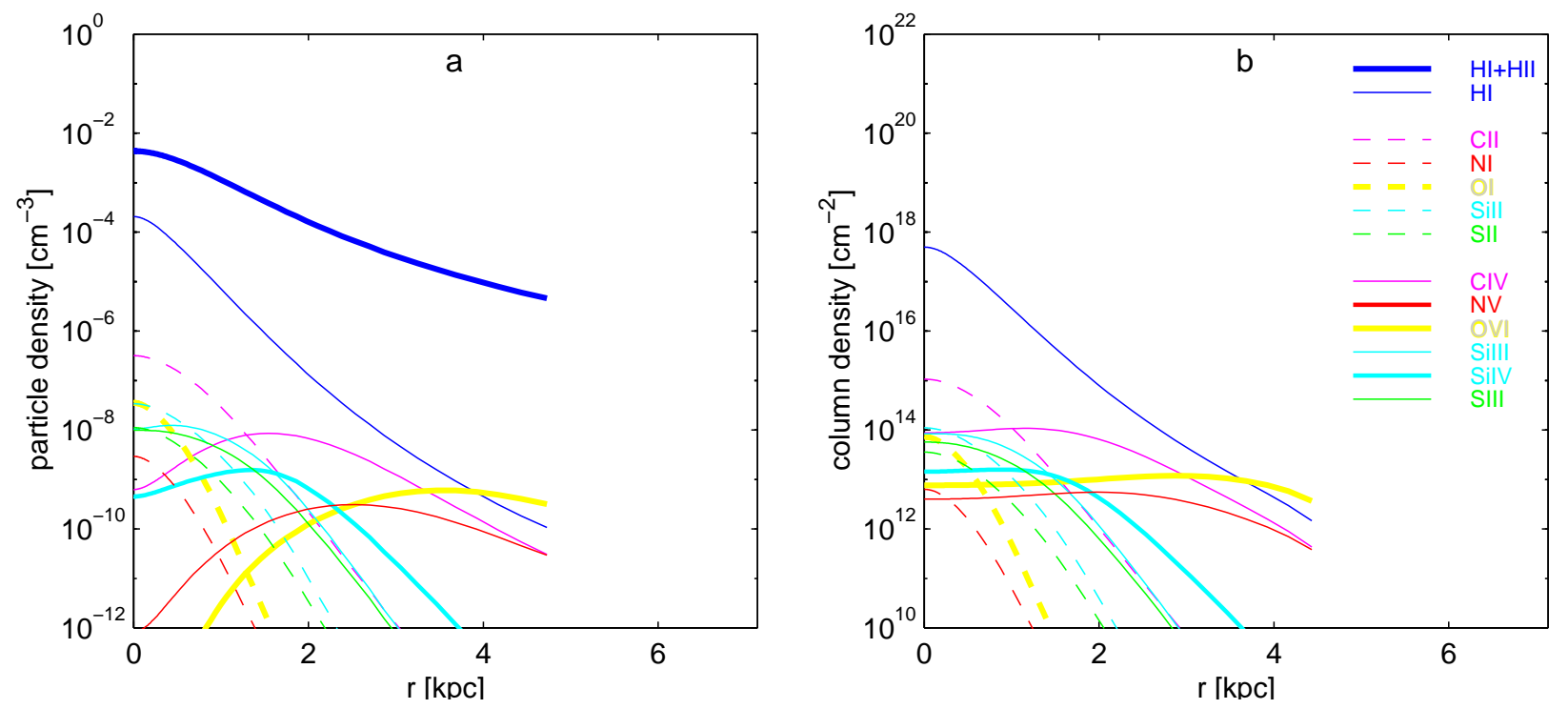

Fig. 12.- Model J: $M_{\text {vir }}=2 \times 10^{9} \mathrm{M}_{\odot}$ median halo, $M_{\text {gas }}=9.6 \times 10^{5} \mathrm{M}_{\odot}, 0.3$ solar metallicity, maximal radiation field, $P / k_{B}=0.1 \mathrm{~cm}^{-3} \mathrm{~K}$. 
Table 1. 0.1 solar metallicity abundances

\begin{tabular}{lc}
\hline \hline Element & $\begin{array}{c}\text { Abundance } \\
(\mathrm{X} / \mathrm{H}) \odot\end{array}$ \\
\hline Carbon & $3.91 \times 10^{-5}$ \\
Nitrogen & $8.53 \times 10^{-6}$ \\
Oxygen & $5.44 \times 10^{-5}$ \\
Silicon & $3.44 \times 10^{-6}$ \\
Sulfur & $1.62 \times 10^{-6}$ \\
\hline
\end{tabular}

Note. - Holweger (2001) 
Table 2. Observed column densities - Sembach et al. (1999)

\begin{tabular}{lllll}
\hline \hline & \multicolumn{3}{c}{ Mrk 509 } & \multicolumn{3}{c}{ PKS 2155-304 } \\
\hline & $-283 \mathrm{~km} \mathrm{~s}^{-1}$ & $-228 \mathrm{~km} \mathrm{~s}^{-1}$ & $-256 \mathrm{~km} \mathrm{~s}^{-1}$ & $-140 \mathrm{~km} \mathrm{~s}^{-1}$ \\
\hline H I & $<4.90 \times 10^{17}$ & $<4.90 \times 10^{17}$ & $<5.37 \times 10^{17}$ & $<5.37 \times 10^{17}$ \\
C II & $4.27_{-2.22}^{+2.19} \times 10^{13}$ & $<3.09 \times 10^{13}$ & & \\
N I & $<3.80 \times 10^{13}$ & $<2.45 \times 10^{13}$ & & \\
Si II & $<1.00 \times 10^{13}$ & $<6.92 \times 10^{12}$ & $<7.24 \times 10^{12}$ & $(5.37 \pm 0.14) \times 10^{13}$ \\
S II & $<8.51 \times 10^{13}$ & $<6.61 \times 10^{13}$ & $<1.58 \times 10^{14}$ & $<1.32 \times 10^{14}$ \\
& & & & \\
C IV & $>1.58 \times 10^{14}$ & $>4.79 \times 10^{13}$ & $(3.16 \pm 0.47) \times 10^{13}$ & $(3.16 \pm 0.47) \times 10^{13}$ \\
N V & $<1.51 \times 10^{13}$ & $<9.55 \times 10^{12}$ & $<1.12 \times 10^{13}$ & $<1.38 \times 10^{13}$ \\
Si III & $>1.55 \times 10^{13}$ & $2.51_{-2.21}^{+2.27} \times 10^{12}$ & & \\
Si IV & $>2.75 \times 10^{13}$ & $<8.51 \times 10^{12}$ & & \\
S III & $<2.29 \times 10^{14}$ & $<2.57 \times 10^{14}$ & & \\
& & & $3.71 \times 10^{12}-1.10 \times 10^{14}$ \\
O VI & $5.75 \times 10^{13}-3.02 \times 10^{14}$ & & \\
\hline
\end{tabular}

Note. - Sembach et al. (1999) (their table 5) - log column densities for two velocity components towards Mrk 509, and two velocity-components toward PKS 2155-304. The error estimates and upper limits are the $2 \sigma$ estimates (The H I upper limits are $4 \sigma$ estimates). The lower limits are due to the possibility of unresolved saturation in the absorption line measurements. OVI values are from Sembach et al. 2000 and 2003. 
Table 3. Observed column densities - Collins et al. (2004)

\begin{tabular}{lllll}
\hline \hline & \multicolumn{1}{c}{ Mrk 509 } & \multicolumn{3}{c}{ PKS 2155-304 } \\
\hline & $-300 \mathrm{~km} \mathrm{~s}^{-1}$ & $-240 \mathrm{~km} \mathrm{~s}^{-1}$ & $-270 \mathrm{~km} \mathrm{~s}^{-1}$ & $-140 \mathrm{~km} \mathrm{~s}^{-1}$ \\
\hline H I & $<4.90 \times 10^{17}$ & $<4.90 \times 10^{17}$ & $1.70_{-0.68}^{+2.38} \times 10^{15}$ & $2.34_{-0.65}^{+1.55} \times 10^{16}$ \\
C II & $4.79_{-0.90}^{+0.97} \times 10^{13}$ & $<2.09 \times 10^{13}$ & $<1.62 \times 10^{13}$ & $6.92_{-1.03}^{+1.79} \times 10^{13}$ \\
C III & & & $3.31_{-0.29}^{+0.24} \times 10^{13}$ & $7.41_{-0.33}^{+0.35} \times 10^{13}$ \\
N I & $<3.39 \times 10^{13}$ & $<2.40 \times 10^{13}$ & $<2.09 \times 10^{13}$ & $<1.74 \times 10^{13}$ \\
N II & & & $<5.13 \times 10^{13}$ & $1.15_{-0.46}^{+0.76} \times 10^{14}$ \\
Si II & $<1.23 \times 10^{13}$ & $<8.51 \times 10^{12}$ & $<5.89 \times 10^{12}$ & $6.46_{-1.33}^{+2.05} \times 10^{12}$ \\
S II & $<1.00 \times 10^{14}$ & $<7.08 \times 10^{13}$ & $<8.71 \times 10^{13}$ & $<6.46 \times 10^{13}$ \\
& & & & \\
C IV & $1.41_{-0.21}^{+0.37} \times 10^{14}$ & $3.39_{-0.44}^{+0.41} \times 10^{13}$ & $3.98_{-0.27}^{+0.38} \times 10^{13}$ & $3.24_{-0.28}^{+0.23} \times 10^{13}$ \\
N V & $<1.74 \times 10^{13}$ & $<1.20 \times 10^{13}$ & $<1.23 \times 10^{13}$ & $<1.20 \times 10^{13}$ \\
O VI & $8.51_{-0.93}^{+0.82} \times 10^{13}$ & $7.76_{-0.68}^{+0.75} \times 10^{13}$ & $3.63_{-0.47}^{+0.44} \times 10^{13}$ & $6.31_{-0.42}^{+0.45} \times 10^{13}$ \\
Si III & $2.04_{-0.18}^{+0.15} \times 10^{13}$ & $2.75_{-0.66}^{+0.71} \times 10^{12}$ & $2.14_{-0.59}^{+0.55} \times 10^{12}$ & $(1.45 \pm 0.07) \times 10^{13}$ \\
Si IV & $3.39_{-0.88}^{+1.86} \times 10^{13}$ & $<3.09 \times 10^{12}$ & $1.82_{-0.56}^{+0.52} \times 10^{12}$ & $5.50_{-0.92}^{+0.96} \times 10^{12}$ \\
S III & $<8.51 \times 10^{13}$ & $<6.03 \times 10^{13}$ & $<4.90 \times 10^{13}$ & $<4.79 \times 10^{13}$ \\
\hline
\end{tabular}

Note. - Column densities for the velocity components towards Mrk 509, and PKS 2155-304. The error estimates are the $1 \sigma$ estimates. The upper limits are $3 \sigma$ limits (The H I upper limits are $4 \sigma$ estimates). The quoted C II, C IV, Si II, and Si IV columns are based on observations of the $\lambda 1036.337, \lambda 1548.203, \lambda 1193.290$, and $\lambda 1393.755$ lines of these ions respectively. 
Table 4. Input Parameters

\begin{tabular}{|c|c|c|c|c|c|c|c|c|}
\hline Model & $\begin{array}{l}M_{\text {vir }} \\
{\left[M_{\odot}\right]}\end{array}$ & $x_{\mathrm{vir}}$ & $\begin{array}{c}v_{s} \\
{\left[\mathrm{~km} \mathrm{~s}^{-1}\right]}\end{array}$ & $\begin{array}{c}r_{s} \\
{[\mathrm{kpc}]}\end{array}$ & $\begin{array}{l}M_{\mathrm{WM}} \\
{\left[M_{\odot}\right]}\end{array}$ & $\begin{array}{c}P_{\mathrm{HIM}} \\
{\left[\mathrm{cm}^{-3} \mathrm{~K}\right]}\end{array}$ & radiation field & $\begin{array}{c}\text { metallicity } \\
\text { [solar] }\end{array}$ \\
\hline \multicolumn{9}{|c|}{ Pressure-confined: } \\
\hline A & $1.0 \times 10^{8}$ & 32.5 & 12 & 0.4 & $1.1 \times 10^{6}$ & 50.0 & Standard & 0.1 \\
\hline B & $3.2 \times 10^{8}$ & $8.15(-4 \sigma)$ & 12 & 2.2 & $7.1 \times 10^{7}$ & 20.0 & Standard & 0.1 \\
\hline \multicolumn{9}{|c|}{ Gravitationally-confined: } \\
\hline$\overline{\mathrm{C}}$ & $2.0 \times 10^{9}$ & 25.5 & 30 & 1.3 & $1.6 \times 10^{7}$ & 1.0 & Standard & 0.1 \\
\hline $\mathrm{D}$ & $2.0 \times 10^{9}$ & 25.5 & 30 & 1.3 & $1.6 \times 10^{7}$ & 0.1 & Standard & 0.1 \\
\hline $\mathrm{E}$ & $2.0 \times 10^{9}$ & 25.5 & 30 & 1.3 & $1.7 \times 10^{7}$ & 0.01 & Standard & 0.1 \\
\hline $\mathrm{F}$ & $2.0 \times 10^{9}$ & 25.5 & 30 & 1.3 & $1.6 \times 10^{7}$ & 1.0 & Maximal & 0.1 \\
\hline G & $2.0 \times 10^{9}$ & 25.5 & 30 & 1.3 & $1.6 \times 10^{7}$ & 0.1 & Maximal & 0.3 \\
\hline $\mathrm{H}$ & $6.2 \times 10^{9}$ & $4.7(-5 \sigma)$ & 30 & 10.2 & $1.3 \times 10^{9}$ & 1.0 & Standard & 0.1 \\
\hline I & $4.9 \times 10^{9}$ & $6.6(-4 \sigma \mathrm{NFW})$ & 30 & 6.7 & $3.1 \times 10^{7}$ & 0.1 & Maximal & 0.3 \\
\hline $\mathrm{J}$ & $2.0 \times 10^{9}$ & 25.5 & 30 & 1.3 & $9.6 \times 10^{5}$ & 0.1 & Maximal & 0.3 \\
\hline
\end{tabular}


Table 5. Results for pressure-confined models

\begin{tabular}{llll}
\hline \hline & & \multicolumn{1}{c}{$\mathrm{A}$} & \multicolumn{1}{c}{$\mathrm{B}$} \\
\hline$N_{H I}$ (peak) & {$\left[\mathrm{cm}^{-2}\right]$} & $4.9 \times 10^{19}$ & $9.7 \times 10^{19}$ \\
$r_{\mathrm{WNM}}$ & {$[\mathrm{kpc}]$} & 0.67 & 3.40 \\
$M_{\mathrm{WNM}}$ & {$\left[\mathrm{M}_{\odot}\right]$} & $3.8 \times 10^{5}$ & $2.0 \times 10^{7}$ \\
& & & \\
$r_{\mathrm{W} / \mathrm{H}}$ & {$[\mathrm{kpc}]$} & 1.30 & 7.15 \\
$U_{\mathrm{W} / \mathrm{H}}$ & & $1.3 \times 10^{-4}$ & $3.5 \times 10^{-4}$ \\
$M_{\mathrm{WIM}}$ & {$\left[\mathrm{M}_{\odot}\right]$} & $7.2 \times 10^{5}$ & $5.1 \times 10^{7}$ \\
& & & \\
\hline & & & \\
$N_{C I V}($ peak$)$ & {$\left[\mathrm{cm}^{-2}\right]$} & $2.6 \times 10^{11}$ & $4.6 \times 10^{12}$ \\
Projected radius at peak $N_{C I V}$ & {$[\mathrm{kpc}]$} & 1.1 & 5.7 \\
& & & \\
Column densities $\left[\mathrm{cm}^{-2}\right]$ at peak $N_{C I V}:$ & & \\
\hline & & & \\
H I & & $3.7 \times 10^{17}$ & $3.6 \times 10^{17}$ \\
C II & & $3.4 \times 10^{14}$ & $5.8 \times 10^{14}$ \\
N I & & $2.7 \times 10^{12}$ & $1.9 \times 10^{12}$ \\
O I & & $2.2 \times 10^{13}$ & $1.6 \times 10^{13}$ \\
Si II & & $2.6 \times 10^{13}$ & $4.1 \times 10^{13}$ \\
S II & & $1.1 \times 10^{13}$ & $1.7 \times 10^{13}$ \\
& & & \\
N V & & $9.5 \times 10^{8}$ & $4.2 \times 10^{10}$ \\
O VI & $3.7 \times 10^{6}$ & $8.7 \times 10^{8}$ \\
Si III & & $1.3 \times 10^{13}$ & $4.9 \times 10^{13}$ \\
Si IV & & $3.7 \times 10^{11}$ & $3.3 \times 10^{12}$ \\
S III & & $7.2 \times 10^{12}$ & $2.6 \times 10^{13}$ \\
\hline & & &
\end{tabular}


Note. - Basic model parameters and column densities of the various metal species at the location of C IV peak column, for pressure-confined CHVC mini-halos. 
Table 6. Results for gravitationally-confined models

\begin{tabular}{|c|c|c|c|c|c|c|c|c|c|}
\hline & & $\mathrm{C}$ & $\mathrm{D}$ & $\mathrm{E}$ & $\mathrm{F}$ & $\mathrm{G}$ & $\mathrm{H}$ & $\mathrm{I}$ & $\mathrm{J}$ \\
\hline$N_{H I}($ peak $)$ & {$\left[\mathrm{cm}^{-2}\right]$} & $1.4 \times 10^{21}$ & $1.4 \times 10^{21}$ & $1.4 \times 10^{21}$ & $1.4 \times 10^{21}$ & $1.3 \times 10^{21}$ & $1.4 \times 10^{21}$ & $1.4 \times 10^{21}$ & $5 \times 10^{17}$ \\
\hline$r_{\mathrm{WNM}}$ & {$[\mathrm{kpc}]$} & 1.2 & 1.2 & 1.2 & 1.2 & 1.2 & 8.5 & 1.5 & 0 \\
\hline$M_{\mathrm{WNM}}$ & {$\left[\mathrm{M}_{\odot}\right]$} & $1.2 \times 10^{7}$ & $1.2 \times 10^{7}$ & $1.2 \times 10^{7}$ & $1.2 \times 10^{7}$ & $1.1 \times 10^{7}$ & $8.5 \times 10^{8}$ & $1.4 \times 10^{7}$ & 0 \\
\hline$r_{\mathrm{W} / \mathrm{H}}$ & {$[\mathrm{kpc}]$} & 4.2 & 7.7 & 13.6 & 4.3 & 7.42 & 24.6 & 12.4 & 4.7 \\
\hline$U_{\mathrm{W} / \mathrm{H}}$ & & $8.9 \times 10^{-3}$ & $9.0 \times 10^{-2}$ & 0.90 & $1.6 \times 10^{-2}$ & 0.15 & $8.8 \times 10^{-3}$ & 0.15 & 0.16 \\
\hline$M_{\mathrm{WIM}}$ & {$\left[\mathrm{M}_{\odot}\right]$} & $4 \times 10^{6}$ & $4 \times 10^{6}$ & $4 \times 10^{6}$ & $4 \times 10^{6}$ & $5 \times 10^{6}$ & $4 \times 10^{8}$ & $1.7 \times 10^{7}$ & $9.6 \times 10^{5}$ \\
\hline$N_{C I V}($ peak $)$ & {$\left[\mathrm{cm}^{-2}\right]$} & $1.3 \times 10^{13}$ & $1.7 \times 10^{13}$ & $1.7 \times 10^{13}$ & $4.7 \times 10^{13}$ & $1.5 \times 10^{14}$ & $7.5 \times 10^{13}$ & $2.6 \times 10^{14}$ & $1.1 \times 10^{14}$ \\
\hline Projected radius at peak $N_{C I V}$ & {$[\mathrm{kpc}]$} & 2.6 & 2.6 & 2.6 & 2.3 & 2.3 & 15.0 & 3.3 & 1.1 \\
\hline \multicolumn{10}{|c|}{ 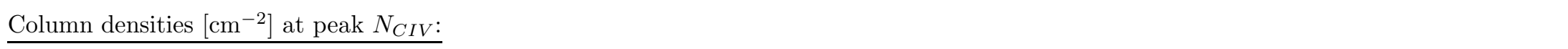 } \\
\hline HI & & $7.3 \times 10^{15}$ & $5.9 \times 10^{15}$ & $5.9 \times 10^{15}$ & $1.8 \times 10^{16}$ & $1.8 \times 10^{16}$ & $4.0 \times 10^{16}$ & $3.0 \times 11^{16}$ & $1.7 \times 10^{16}$ \\
\hline CII & & $2.1 \times 10^{13}$ & $1.7 \times 10^{13}$ & $1.7 \times 10^{13}$ & $2.1 \times 10^{13}$ & $6.5 \times 10^{13}$ & $1.1 \times 10^{14}$ & $1.1 \times 10^{14}$ & $6.6 \times 10^{13}$ \\
\hline NI & & $6.9 \times 10^{9}$ & $4.4 \times 10^{9}$ & $4.5 \times 10^{9}$ & $5.9 \times 10^{9}$ & $1.8 \times 10^{10}$ & $3.4 \times 10^{10}$ & $3.0 \times 10^{10}$ & $2.3 \times 10^{10}$ \\
\hline OI & & $3.7 \times 10^{10}$ & $2.3 \times 10^{10}$ & $2.3 \times 10^{10}$ & $3.8 \times 10^{10}$ & $1.2 \times 10^{11}$ & $1.9 \times 10^{11}$ & $2.0 \times 10^{11}$ & $1.6 \times 10^{11}$ \\
\hline SiII & & $1.1 \times 10^{12}$ & $8.3 \times 10^{11}$ & $8.4 \times 10^{11}$ & $2.1 \times 10^{12}$ & $6.2 \times 10^{12}$ & $5.6 \times 10^{12}$ & $1.0 \times 10^{13}$ & $6.4 \times 10^{12}$ \\
\hline SII & & $4.8 \times 10^{11}$ & $3.7 \times 10^{11}$ & $3.7 \times 10^{11}$ & $6.1 \times 10^{11}$ & $1.8 \times 10^{12}$ & $2.4 \times 10^{12}$ & $3.1 \times 10^{12}$ & $1.9 \times 10^{12}$ \\
\hline $\mathrm{NV}$ & & $3.4 \times 10^{11}$ & $9.0 \times 10^{11}$ & $1.0 \times 10^{12}$ & $1.4 \times 10^{12}$ & $7.1 \times 10^{12}$ & $2.0 \times 10^{12}$ & $1.2 \times 10^{13}$ & $4.7 \times 10^{12}$ \\
\hline OVI & & $2.1 \times 10^{11}$ & $1.9 \times 10^{12}$ & $3.0 \times 10^{12}$ & $8.6 \times 10^{11}$ & $1.3 \times 10^{13}$ & $1.3 \times 10^{12}$ & $2.2 \times 10^{13}$ & $8.2 \times 10^{12}$ \\
\hline SilII & & $7.0 \times 10^{12}$ & $6.1 \times 10^{12}$ & $6.1 \times 10^{12}$ & $1.1 \times 10^{13}$ & $3.3 \times 10^{13}$ & $3.7 \times 10^{13}$ & $5.6 \times 10^{13}$ & $3.0 \times 10^{13}$ \\
\hline SiIV & & $2.8 \times 10^{12}$ & $2.9 \times 10^{12}$ & $2.9 \times 10^{12}$ & $6.8 \times 10^{12}$ & $2.0 \times 10^{13}$ & $1.6 \times 10^{13}$ & $3.5 \times 10^{13}$ & $1.5 \times 10^{13}$ \\
\hline SIII & & $3.7 \times 10^{12}$ & $3.2 \times 10^{12}$ & $3.3 \times 10^{12}$ & $5.7 \times 10^{12}$ & $1.7 \times 10^{13}$ & $2.0 \times 10^{13}$ & $2.9 \times 10^{13}$ & $1.6 \times 10^{13}$ \\
\hline
\end{tabular}


Note. - Same as Table 5 but for dwarf-galaxy scale mini-halos. 